\title{
Prevalence, incidence, and trends of childhood overweight/obesity in Sub- Saharan Africa: a systematic scoping review
}

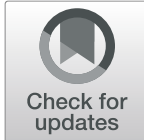

Frederick Inkum Danquah", Monica Ansu-Mensah', Vitalis Bawontuo ${ }^{1,2}$, Matilda Yeboah ${ }^{1}$ and Desmond Kuupiel ${ }^{1,2,3^{*}}$ (i)

\begin{abstract}
Background: The growing burden of non-communicable diseases (NDC), particularly in low-and middle-income countries, poses a significant threat to global health. Obesity and overweight constitute major risk factors of NCDs such as heart diseases, diabetes, and kidney disease, and as a result, contribute significantly to the development of chronic morbidities, reduced quality of life, and increased risk of premature death. This study described evidence on the prevalence, incidence, and trends of childhood overweight and obesity in sub-Sahara Africa (SSA).

Methods: We conducted a systematic scoping review employing the Arksey and O'Malley framework, Levac et al. recommendations, and the Joanna Briggs Institute guidelines. To obtain relevant published articles for this review, we performed a comprehensive keywords search in PubMed, Google Scholar, Web of Science, and CINAHL via EBSCOhost platform for studies published between 2009 and June 2019. Guided by the eligibility criteria, title and abstracts, as well as the full-text articles were independently screened in parallel by two investigators. All relevant data were independently extracted by two investigators using a piloted form designed in Microsoft and thematic analysis conducted.

Results: Of the 81 included studies obtained from 250,148 potentially eligible articles, the majority (25) conducted in South Africa followed by 18 in Nigeria. Six studies were conducted in Ethiopia (6), Tanzania (5), Kenya (4), Cameroon (4), Ghana (3), Uganda (2), Mozambique (2), and Sudan (2). One study each was conducted in Botswana, Gambia, Lesotho, Mauritius, Seychelles, Togo, and Zimbabwe. The remaining three articles were multi-country studies. Most (81.5\%) of the included studies were cross-sectional surveys and the majority (79) focused on both male and female participants. The majority (80/ 81) of the included studies reported on the prevalence of childhood overweight/obesity, 8 on the trends of childhood overweight/obesity, and one presented evidence on the incidence of childhood overweight and obesity in SSA.

Conclusion: This review demonstrates limited studies on childhood overweight/obesity in most SSA countries although the included studies suggest an increasing burden. Considering the consequences of childhood obesity, there is a need for more primary researches to inform policies decision and implementation to halt the rise of childhood obesity/overweight in SSA.
\end{abstract}

Keywords: Childhood, Obesity, Overweight, Prevalence, Incidence, Trends, Sub-Sahara Africa

\footnotetext{
*Correspondence: desmondkuupiel98@hotmail.com; KuupielD@ukzn.ac.za

'Department of Public Health, Faculty of Health and Allied Sciences, Catholic

University College of Ghana, Fiapre, Sunyani, Ghana

${ }^{2}$ Research for Sustainable Development Consult, Sunyani, Ghana

Full list of author information is available at the end of the article
}

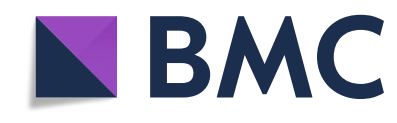

(c) The Author(s). 2020 Open Access This article is licensed under a Creative Commons Attribution 4.0 International License, which permits use, sharing, adaptation, distribution and reproduction in any medium or format, as long as you give appropriate credit to the original author(s) and the source, provide a link to the Creative Commons licence, and indicate if changes were made. The images or other third party material in this article are included in the article's Creative Commons licence, unless indicated otherwise in a credit line to the material. If material is not included in the article's Creative Commons licence and your intended use is not permitted by statutory regulation or exceeds the permitted use, you will need to obtain permission directly from the copyright holder. To view a copy of this licence, visit http://creativecommons.org/licenses/by/4.0/. The Creative Commons Public Domain Dedication waiver (http://creativecommons.org/publicdomain/zero/1.0/) applies to the data made available in this article, unless otherwise stated in a credit line to the data. 


\section{Background}

Globally, the burden of non-communicable diseases (NCDs) is rising and has been recognized as a global health issue [1]. NCDs are reported to account for over half of all global health problems [2] and contribute substantially to morbidity and mortality [3]. The Global Burden of Disease 2013 study report indicated that NCDs were responsible for about $70 \%$ (38.3million) of the 54.9 million deaths worldwide of which about $80 \%$ occurred in low-and middle-income countries (LMICs) [2, 4]. Nutritional disorders such as obesity, overweight, underweight, and stunting in childhood and adolescence are associated with adverse health consequences throughout the life-span [5]. The World Health Organization (WHO) defines obesity and overweight as an abnormal or excessive accumulation of body fat that may impair health [6].

Obesity has been described as one of the greatest health challenges and determinants for many chronic diseases and psychosocial problems and is the fifth leading cause of mortality globally [7]. Obesity and overweight result from a complex interplay of genetic, metabolic, behavioral, cultural, and environmental factors [8, 9]. The epidemic of obesity is mostly driven by the global food system including the increased supply of cheap, palatable, energy-dense foods; improved distribution systems to make food much more accessible and convenient; and more persuasive and pervasive food marketing [10]. The body mass index (BMI) is a simple index of weight-for-height defined as a person's weight in kilograms divided by the square of his height in meters $\left(\mathrm{kg} / \mathrm{m}^{2}\right)$ which is commonly used to classify overweight and obesity $[11,12]$. BMI percentiles for age and sex have been developed for diagnosing children older than 2 years of age $[13,14]$, where children with BMI above the 85th and 95th percentiles are classified as having a higher risk of overweight and obesity respectively $[15,16]$.

Obesity is one of the major risk factors which predisposes children to the development of non-communicable diseases such as heart disease, diabetes, cancers, and kidney disease, and as a result, contributes significantly to the development of chronic morbidities, increased risk of premature death [17, 18], and reduced quality of life [19]. Muthuri et al. [20] asserted that the health risks associated with obesity and overweight are particularly problematic in children due to the potential for long term health consequences. Obese children are more likely to experience breathing difficulties, increased risk of fractures, and hypertension which are early markers of cardiovascular disease, as well as insulin resistance and psychological problems, in addition to higher chances of premature death and disability in adulthood [6].

Lobstein and Jackson-Leach in 2016 estimated that by 2025 some 268 million children aged 5-17 years globally may be overweight, including 91 million obese based on the assumption that no policy interventions prove effective at changing this trend [21]. Available evidence suggests that the situation in sub-Saharan Africa (SSA) is likely to be worsened by the current nutrition and physical activity transition characterized by increased use of energy-saving devices, availability of cheap highcalorie dense foods, and limited participation in physical activity generally $[22,23]$. Moreover, many studies have stressed the negative influence of some sociocultural beliefs in which obesity and overweight are revered and seen as a sign of prestige, good life, and economic value $[22,24-26]$. Other studies have also pointed to an obvious link between higher NCD/obesity-related mortalities and increasing socioeconomic development among SSA countries [27]. Despite this, to date, no study has mapped evidence on childhood obesity and identified research gaps to the best of our knowledge. We, therefore, sought to systematically search and examine literature and describe the evidence on the prevalence, incidence, and trends of childhood overweight and obesity in SSA.

\section{Methods}

We conducted a systematic scoping review employing the Arksey and O'Malley framework, Levac et al. recommendations, and the Joanna Briggs Institute guidelines [28-30]. This study forms part of a larger study titled: "Mapping evidence on the burden and distribution of childhood obesity in sub-Saharan Africa". However, the present study aimed at presenting evidence on the prevalence, incidence, and trends of childhood obesity in SSA. A detailed description of this study methods has been published elsewhere [31]. The Preferred Reporting Items for Systematic reviews and Meta-Analyses extension for Scoping Reviews (PRISMA-ScR) Checklist was followed to report this study (Supplementary file 1).

\section{Identifying the research question}

This scoping review sought to answer the following question: What is the evidence on the prevalence, incidence, and trends of childhood obesity in SSA? To determine the eligibility of the review question for this study, the population, exposure, and outcome (PEO) mnemonic was used (Table 1).

\section{Identifying relevant studies}

To obtain relevant published articles for this review, we conducted an exhaustive keywords search in PubMed, Google Scholar, Web of Science databases, and CINAHL via EBSCOhost platform. The database search was conducted between May 2019 and June 2019. In consultation with an experienced Librarian, we developed a comprehensive keywords search strategy to enable identification of all relevant articles as found in the published protocol [31]. Boolean terms, AND/OR were used to separate the keywords. Medical Subject Heading (MeSH) 
Table 1 PEO framework for determining the eligibility of the scoping review question

\begin{tabular}{ll}
\hline P-Population & Children (persons aged from $\mathbf{2}$ to $\mathbf{1 8}$ years) \\
\hline E-Exposure & Overweight (children with $\mathrm{BMI} \geq 85$ th percentile) \\
& Obesity BMI $\left(\geq 95\right.$ th percentile or $\left.\geq 35 \mathrm{~kg} / \mathrm{m}^{2}\right)$ \\
O-Outcome & Prevalence \\
& Incidence \\
& Trends/distribution \\
\hline
\end{tabular}

terms were also included in the search. The date was limited from 2009 to 2019, and language and study design limitations were removed during the search. We also searched the reference list of included studies for possible relevant articles. Supplementary file 2 presents the complete search strategy in the electronic databases.

\section{Eligibility criteria and study selection}

This study included articles reporting evidence on obesity/ overweight in children aged from 2 to 18 years, articles focused on incidence/prevalence/trends of childhood obesity/ overweight, and studies conducted in SSA. Also, only studies published between 2009 to 2019 and in English language were included. Furthermore, this review included only quantitative studies utilising internationally recognized criteria for assessing body composition. However, this study excluded studies reporting evidence on obesity prevalence, incidence and trends among people aged less than 2 years or more than 18 years, risk factors of childhood obesity, studies focusing on knowledge of childhood obesity, articles published in French, and systematic reviews and meta-analyses. Studies conducted in countries not classified among SSA as well as in high-income countries were also excluded.

To reduce selection bias, FID and MAM conducted a comprehensive title search in the databases guided by the eligibility criteria. All potentially eligible studies were imported into Mendeley Desktop that was created for this review and duplicates removed. Again, FID and MAM independently screened the abstracts and fulltexts using the inclusion and exclusion criteria. Discrepancies among the reviewers following abstract screening were resolved through discussions to build consensus. However, DK addressed the discrepancies between FID and MAM at the full-text screening stage. Subsequently, the inter-rater agreement (Cohen's kappa coefficient, $\mathrm{k}$ statistic) between the reviewers was calculated after the full-text screening using Stata version 14. An adapted PRISMA (Preferred Reporting Items for Systematic Reviews and Meta-Analysis) flow diagram was employed to document the study selection process [32].

\section{Charting data}

FID and MY independently extracted data using a tabular piloted form designed in Microsoft excel and DK resolved the discrepancies. Elements of the data extraction form included; author and date, study design, country and setting, sample size, age, gender, and significant outcomes reported (prevalence, incidence, and trend). We also extracted the criteria used for the assessment of body composition.

\section{Collating and summarizing the results}

Following the data extraction, a thematic content analysis was conducted. A narrative approach was used to report the findings from the included studies. The findings from the included studies were summarised and manually coded into the following themes: prevalence, incidence, and trends of childhood obesity.

\section{Results}

In all, 250,148 articles were obtained from all databases during the initial search, of which 959 titles met the eligibility criteria at the title screening stage. A total of 593 titles were identified as duplicates and removed using Mendeley Desktop which was used to compile all the eligible titles from the databases. Subsequently, 366 articles underwent abstract screening of which 112 met the inclusion criteria and were included for full-text screening. A total of 31 articles were excluded following the full-text screening stage, and the remaining 81 articles were included for data extraction (Fig. 1). The inter-rater agreement at the full-text screening (Kappa statistic $=0.75, p<$ 0.01 ) and data extraction (Kappa statistic $=0.87, p<0.01$ ) stages shows there was a moderate to a substantial level of agreement respectively. Of the 31 articles excluded at the full-text screening stage, 17 did not report any of this study's outcomes of interest [3, 33-48], 7 were review studies [20, 49-54], 4 were published in French though their titles and abstracts were in English [55-58], 2 reported evidence outside this study's population age range $[59,60]$, and 1 study was not conducted in of SSA [61].

\section{Characteristics of the included studies}

Out of the 81 included studies, $66(81.5 \%)$ were crosssectional surveys $[16,24,62-125]$; 8 (9.9\%) were secondary data analysis [126-133]; 4 (4.9\%) were longitudinal studies $[15,134-136]$; one $(1.2 \%)$ was a national demographic health survey (DHS) [137]; one $(1,2 \%)$ intervention trial [138]; and one (1.2\%) case study [139]. The 81 included studies were conducted in 20 SSA countries of which 17 were single country studies, and 3 multicountry studies. Of the 81 included studies, the majority (25) were conducted in South Africa followed by Nigeria with 18 studies. One of the multi-country studies reported evidence 7 countries of which 4 (Benin, Ghana, Mauritania, and Malawi) were SSA countries [111]. The remaining two multi-country studies reported evidence from two countries each. That is, Kenya and South 


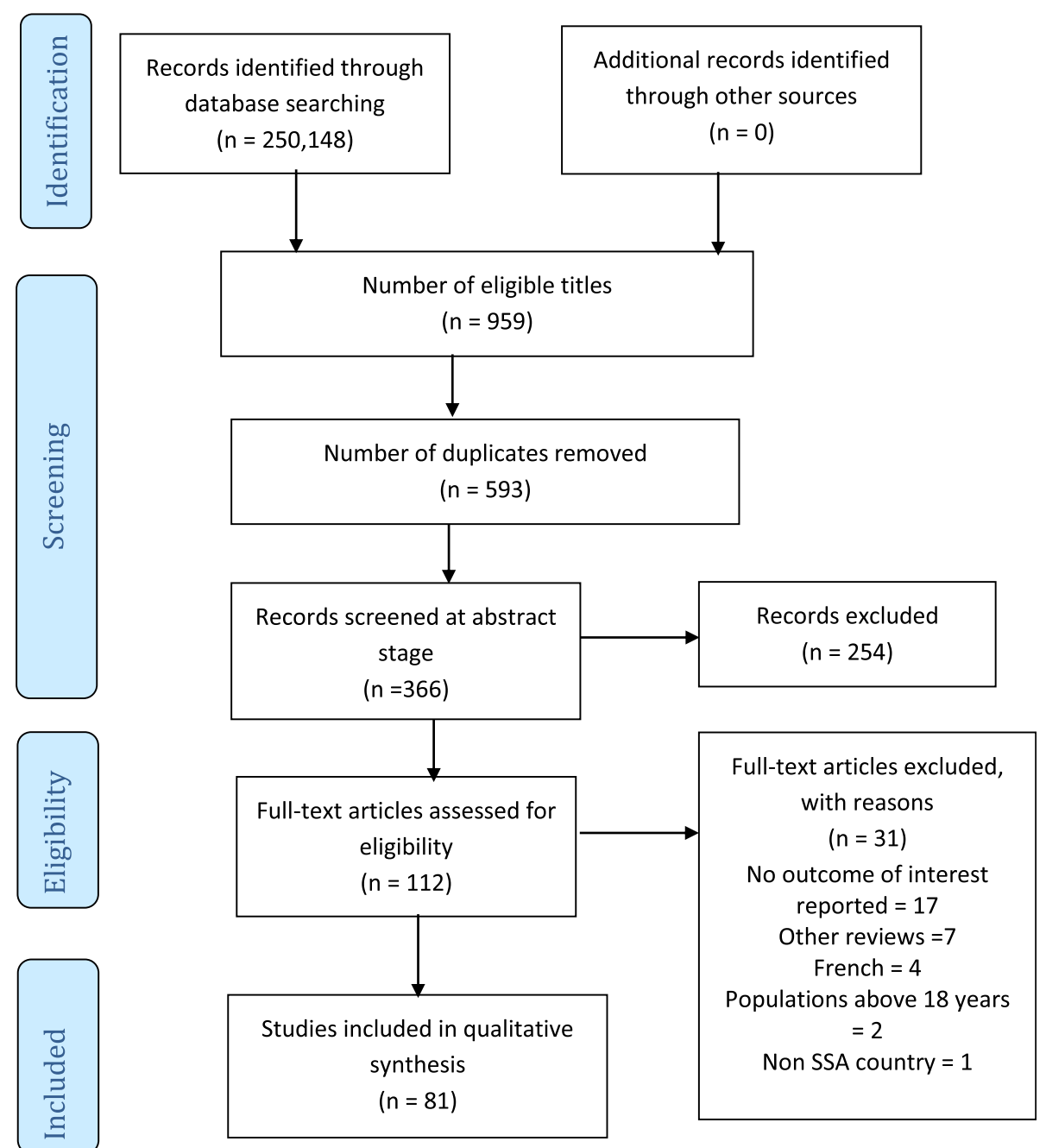

Fig. 1 PRISMA chart showing results of literature search and study selection

Africa [110], and Ghana and Uganda [128]. Figure 2 illustrated the distribution of the included studies per country. (Fig. 2).

About 47\% (38/81) of the studies were conducted in urban settings; $33.3 \%(27 / 81)$ in both rural and urban areas; $7 \%(6 / 81)$ in rural settings; $3.7 \%(3 / 81)$ in semiurban settings; $2.5 \%(2 / 81)$ in urban and suburban settings; and $1.2 \%(1 / 81)$ in a peri-urban setting. In about $5 \%(4 / 81)$ of the included studies, there was no indication of the study setting. Most of the included studies $97.5 \%(79 / 81)$ reported on both males and females and $2.5 \%(2 / 81)$ reported evidence on only females. There was no study conducted with only male participants.

Approximately 96\% (78/81) of the included studies reported evidence on children older than 5 years whilst about $4 \%(3 / 81)$ reported on children under 5 years. The sample size of the included studies ranged from 56 [139] to 24,391 [63] participants. Of the 81 studies, 39 (48.2\%) utilized WHO reference criteria, 26 (32.1\%) employed
Cole et al. and international obesity task force (IOTF) cut-offs, 6 (7.4\%) used BMI cut-of-points, and 3 (3.7\%) utilized United States of America Centers for Disease Control and Prevention BMI growth charts. Seven (8.6\%) of the 81 included studies compared two or more of the assessment criteria (Table 2).

\section{Study findings}

In all, 80 of the 81 included studies reported findings on the prevalence of childhood overweight/obesity, 8 on trends of childhood overweight/obesity, and one reported on the incidence of childhood overweight/ obesity.

\section{Prevalence of childhood overweight/obesity}

Most $(n=80)$ of the included studies reported evidence on the prevalence of obesity/overweight among children and adolescents. Armstrong et.al. in South Africa involving 24,391 school children aged from 8 to 11 years, 


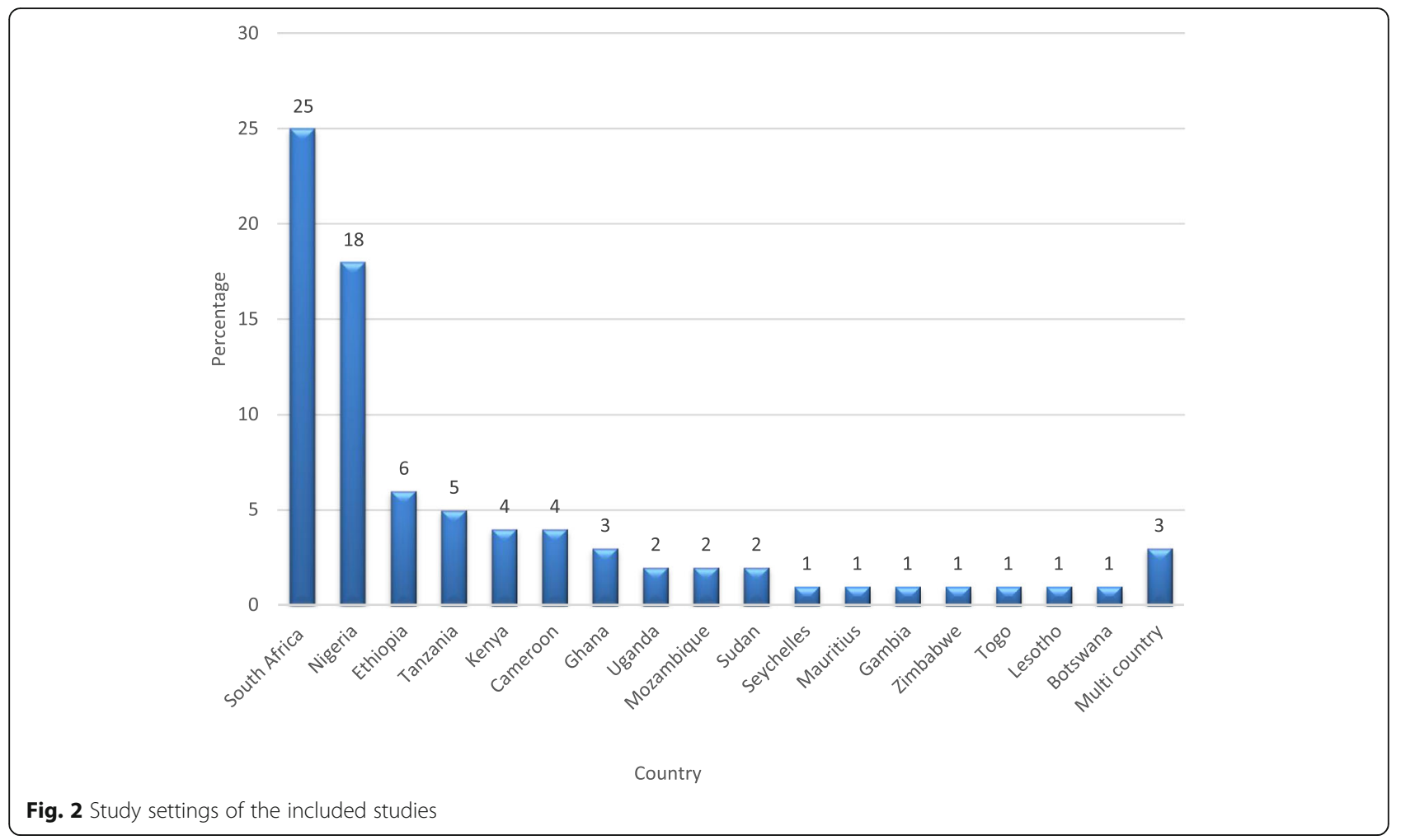

reported 13 and 3.3\% childhood prevalence of overweight and obesity respectively in 2004 [63]. Armstrong et.al. noted that these findings were much higher than the baseline values of 1.2 and $0.2 \%$ prevalence of childhood overweight and obesity respectively in 1994 [63]. Again, Armstrong et.al [64]. study involving school children aged 6 to 13 years old reported a combined overweight and obesity prevalence of $15.4 \%(1564 / 10,285)$. Craig et al. [65] study compared different international standards of assessing body composition in rural KwaZulu-Natal. Using WHO 2007 reference criteria, the combined overweight/obesity prevalence was 9.2, 8.1, and $8.0 \%$ in males compared to $13.6,13.4$, and $25.8 \%$ in females aged 7,11 , and 15 years respectively [65]. These findings however differed substantially when Cole et al. and IOFT cut-offs were used, with reported combined childhood obesity and overweight prevalence of 3.2, 5.2, and $6.1 \%$ in males compared to $9.2,9.7$, and $22.7 \%$ in females within the respective age $(7,11,15$ years) distributions [65]. Kimani-Murage et al. in rural Mpumalanga Province, found a prevalence of overweight/obesity of $4 \%$ in boys and $15 \%$ in girls among 1848 adolescents aged 10 to 20 years [66]. Ngwenya et al. studied 175 adolescents from the urban city of Tshwane and identified $15.4 \%(n=27)$ overweight and $8.6 \%(n=15)$ obesity prevalence [67]. In the Western Cape, Negash et al. reported an overall prevalence of overweight/obesity of $22.9 \%$ with girls having significantly higher levels (19.7\%/
9.1\%) than boys $(9.4 \% / 4.5 \%)$ and Whites learners being more overweight $(20.7 \%)$ while Coloured learners were more obese (7.5\%) [68]. Similarly, Feeley et al. recorded a combined overweight/obesity prevalence of 8.1 and $27.0 \%$ in males and females respectively, while Ginsburg et al. reported a combined overweight/obesity prevalence of 8.0 and $25.0 \%$ for males and females respectively, albeit with different sample sizes [134, 135]. Pretorius et al. and Symington et al. reported 27.3 and $12.0 \%$ prevalence of overweight/obesity in their respective studies of rural-urban populations in South Africa $[69,130]$. Using WHO growth standards, Lesiapeto et al. identified a combined overweight/obesity prevalence of $16.1 \%$ among 2485 children under 5 years in two rural districts of South Africa [126]. Additionally, a case study conducted by Mokabane et al. among 56 black girls aged 13 to 19 years in a peri-urban area of Limpopo Province identified a $12.5 \%$ overweight and $3.6 \%$ obesity prevalence [139].

Of the 18 studies conducted in Nigeria, 17 provided evidence on childhood overweight/obesity prevalence [70-84, 86, 87]. A study by Adegoke et al. in 2009 aimed to determine the nutritional status of children aged from 6 to18 years in Ile-Ife observed a prevalence of $0.3 \%$ (2/ 720 ) obesity (both being female), and $2.8 \%(20 / 720)$ overweight (of which 15 (85\%) were female) [70]. Omuemu et al. study in an urban city of Edo State reported 5.7\% overweight/obesity [71]; Opara et al. reported 11.1 and $0.2 \%$ of overweight/obesity respectively 
Table 2 Characteristics and findings of the included studies

\begin{tabular}{|c|c|c|c|c|c|c|c|c|c|c|c|}
\hline & $\begin{array}{l}\text { Author \& } \\
\text { date }\end{array}$ & $\begin{array}{l}\text { Study } \\
\text { design }\end{array}$ & Country & Setting & $\begin{array}{l}\text { Sample } \\
\text { size }\end{array}$ & $\begin{array}{l}\text { Age } \\
\text { range } \\
\text { (years) }\end{array}$ & Gender & $\begin{array}{l}\text { Outcome } \\
\text { reported }\end{array}$ & $\begin{array}{l}\text { Prevalence } \\
\text { of } \\
\text { overweight }\end{array}$ & $\begin{array}{l}\text { Prevalence } \\
\text { of obesity }\end{array}$ & $\begin{array}{l}\text { Criteria for } \\
\text { assessment } \\
\text { of body } \\
\text { composition }\end{array}$ \\
\hline 1 & $\begin{array}{l}\text { Armstrong } \\
\text { et al., } 2011 \\
\text { [63] }\end{array}$ & $\begin{array}{l}\text { Cross } \\
\text { sectional }\end{array}$ & South Africa & $\begin{array}{l}\text { Rural, } \\
\text { urban }\end{array}$ & 24,391 & $8-11$ & $\begin{array}{l}\text { Male, } \\
\text { female }\end{array}$ & $\begin{array}{l}\text { Prevalence, } \\
\text { trend }\end{array}$ & $13.0 \%$ & $3.3 \%$ & IOTF \\
\hline 2 & $\begin{array}{l}\text { Armstrong } \\
\text { et al., } 2017 \\
\text { [64] }\end{array}$ & $\begin{array}{l}\text { Cross- } \\
\text { sectional }\end{array}$ & South Africa & $\begin{array}{l}\text { Rural, } \\
\text { urban }\end{array}$ & 10,285 & $6-13$ & $\begin{array}{l}\text { Male, } \\
\text { female }\end{array}$ & prevalence & $15.4 \%$ & & IOTF \\
\hline 3 & $\begin{array}{l}\text { Baumgartner } \\
\text { et al., } 2013 \\
{[138]}\end{array}$ & $\begin{array}{l}\text { Placebo- } \\
\text { controlled, } \\
\text { double- } \\
\text { blind inter- } \\
\text { vention trial. }\end{array}$ & South Africa & $\begin{array}{l}\text { Rural, } \\
\text { urban }\end{array}$ & 321 & $6-11$ & $\begin{array}{l}\text { Male, } \\
\text { female }\end{array}$ & Prevalence & $28 \%$ & & WHO \\
\hline 4 & $\begin{array}{l}\text { Craig et al., } \\
2013 \text { [65] }\end{array}$ & $\begin{array}{l}\text { Cross- } \\
\text { sectional }\end{array}$ & South Africa & Rural & 1519 & $7-15$ & $\begin{array}{l}\text { Male, } \\
\text { female }\end{array}$ & Prevalence & \multicolumn{2}{|c|}{$\begin{array}{l}\text { 9.2, } 8.1 \text {, and } 8.0 \% \text { in males } \\
\text { compared to } 13.6,13.4, \text { and } \\
25.8 \% \text { in females aged } 7,11 \text {, } \\
\text { and } 15 \text { years respectively } \\
\text { using WHO } 2007 \text { reference } \\
\text { criteria } \\
9.2,8.1 \text {, and } 8.0 \% \text { in males } \\
\text { compared to } 13.6,13.4, \text { and } \\
25.8 \% \text { in females aged } 7,11 \text {, } \\
\text { and } 15 \text { years respectively } \\
\text { using Cole et al. and IOFT }\end{array}$} & $\begin{array}{l}\text { WHO } \\
\text { IOTF } \\
\text { NCHS/WHO }\end{array}$ \\
\hline 5 & $\begin{array}{l}\text { Feeley et al., } \\
2013[134]\end{array}$ & Longitudinal & South Africa & Urban & 1298 & $13-17$ & $\begin{array}{l}\text { Male, } \\
\text { female }\end{array}$ & $\begin{array}{l}\text { Prevalence, } \\
\text { trend }\end{array}$ & \multicolumn{2}{|c|}{$\begin{array}{l}8.1 \% \text { (Males) and } 27.0 \% \\
\text { (Females) }\end{array}$} & WHO \\
\hline 6 & $\begin{array}{l}\text { Ginsburg } \\
\text { et al., } 2013 \\
\text { [135] }\end{array}$ & Longitudinal & South Africa & Urban & 1613 & 15 & $\begin{array}{l}\text { Male, } \\
\text { female }\end{array}$ & Prevalence & \multicolumn{2}{|c|}{$\begin{array}{l}8.0 \% \text { (Males) and } 25.0 \% \\
\text { (Females) }\end{array}$} & IOTF \\
\hline 7 & $\begin{array}{l}\text { Kimani- } \\
\text { Murage et al., } \\
2010 \text { [115] }\end{array}$ & $\begin{array}{l}\text { Cross- } \\
\text { sectional }\end{array}$ & South Africa & Rural & 3511 & $1-20$ & $\begin{array}{l}\text { Male } \\
\text { female }\end{array}$ & Prevalence & \multicolumn{2}{|c|}{$\begin{array}{l}18 \% \text { in females compared to } \\
4 \% \text { in males }\end{array}$} & IOTF \\
\hline 8 & $\begin{array}{l}\text { Kimani- } \\
\text { Murage et al., } \\
2011 \text { [66] }\end{array}$ & $\begin{array}{l}\text { Cross- } \\
\text { sectional }\end{array}$ & South Africa & Rural & 1848 & $10-20$ & $\begin{array}{l}\text { Male, } \\
\text { female }\end{array}$ & Prevalence & \multicolumn{2}{|c|}{ 4\% Boys) and 15\% (Girls) } & IOTF \\
\hline 9 & $\begin{array}{l}\text { Kruger et al., } \\
2011[112]\end{array}$ & $\begin{array}{l}\text { Cross- } \\
\text { sectional }\end{array}$ & South Africa & $\begin{array}{l}\text { Rural, } \\
\text { urban }\end{array}$ & 2157 & $1-9$ & $\begin{array}{l}\text { Male, } \\
\text { female }\end{array}$ & $\begin{array}{l}\text { Prevalence, } \\
\text { trend }\end{array}$ & $10 \%$ & $4 \%$ & WHO \\
\hline 10 & $\begin{array}{l}\text { Lesiapeto } \\
\text { et al., } 2016 \\
{[126]}\end{array}$ & $\begin{array}{l}\text { Secondary } \\
\text { analysis }\end{array}$ & South Africa & Rural & 2485 & $\begin{array}{l}\text { Under } \\
5\end{array}$ & $\begin{array}{l}\text { Male, } \\
\text { female }\end{array}$ & Prevalence & \multicolumn{2}{|l|}{$16.1 \%$} & WHO \\
\hline 11 & $\begin{array}{l}\text { Lundeen et al., } \\
2015 \text { [127] }\end{array}$ & $\begin{array}{l}\text { Secondary } \\
\text { analysis }\end{array}$ & South Africa & Urban & 1172 & $1-18$ & $\begin{array}{l}\text { Male, } \\
\text { female }\end{array}$ & $\begin{array}{l}\text { Prevalence, } \\
\text { incidence, } \\
\text { trend }\end{array}$ & $\begin{array}{l}\text { Boys }=19.1 \\
16.4,9.9,7.8, \\
5.7 \% \text { and } \\
\text { Girls }=19.1 \\
12.2,14.7 \\
17.8, \text { and } \\
19.1 \% \text { at } 1-2, \\
4-8,11-12 \\
13-15, \text { and } \\
16-18 \text { years } \\
\text { respectively. }\end{array}$ & $\begin{array}{l}\text { Boys }=8.8, \\
3.0,6.0,4.4 \%, \\
2.5 \text { and } \\
\text { Girls }=8.1 \\
3.1,6.4,7.3 \\
\text { and } 7.9 \% \text { at } \\
1-2,4-8,11- \\
12,13-15, \\
\text { and } 16-18 \\
\text { years } \\
\text { respectively. }\end{array}$ & WHO \\
\hline 12 & $\begin{array}{l}\text { Meko et al., } \\
2015 \text { [116] }\end{array}$ & $\begin{array}{l}\text { Cross- } \\
\text { sectional }\end{array}$ & South Africa & Urban & 415 & $13-15$ & $\begin{array}{l}\text { Male, } \\
\text { female }\end{array}$ & Prevalence & \multicolumn{2}{|l|}{$6 \%$} & WHO \\
\hline 13 & $\begin{array}{l}\text { Mokabane } \\
\text { et al., } 2014 \\
\text { [139] }\end{array}$ & Case study & South Africa & $\begin{array}{l}\text { Peri- } \\
\text { urban }\end{array}$ & 56 & $13-19$ & Female & Prevalence & $12.5 \%$ & $3.6 \%$ & BMI \\
\hline 14 & $\begin{array}{l}\text { Moselakgomo } \\
\text { et al., } 2017 \\
\text { [117] }\end{array}$ & $\begin{array}{l}\text { Cross- } \\
\text { sectional }\end{array}$ & South Africa & Rural & 1361 & $9-13$ & $\begin{array}{l}\text { Male, } \\
\text { female }\end{array}$ & Prevalence & $\begin{array}{l}\text { Boys }=9.9 \% \\
\text { (CDC } \\
\text { classification) } \\
\text { and } 2.6 \%\end{array}$ & $\begin{array}{l}\text { Boys }=5.46 \% \\
\text { (CDC } \\
\text { classification) } \\
\text { and } 0.7 \%\end{array}$ & $\begin{array}{l}\text { CDC } \\
\text { IOTF }\end{array}$ \\
\hline
\end{tabular}


Table 2 Characteristics and findings of the included studies (Continued)

\begin{tabular}{|c|c|c|c|c|c|c|c|c|c|c|c|}
\hline & $\begin{array}{l}\text { Author \& } \\
\text { date }\end{array}$ & $\begin{array}{l}\text { Study } \\
\text { design }\end{array}$ & Country & Setting & $\begin{array}{l}\text { Sample } \\
\text { size }\end{array}$ & $\begin{array}{l}\text { Age } \\
\text { range } \\
\text { (years) }\end{array}$ & Gender & $\begin{array}{l}\text { Outcome } \\
\text { reported }\end{array}$ & $\begin{array}{l}\text { Prevalence } \\
\text { of } \\
\text { overweight }\end{array}$ & $\begin{array}{l}\text { Prevalence } \\
\text { of obesity }\end{array}$ & $\begin{array}{l}\text { Criteria for } \\
\text { assessment } \\
\text { of body } \\
\text { composition }\end{array}$ \\
\hline & & & & & & & & & $\begin{array}{l}\text { (IOTF criteria) } \\
\text { Girls = } 10.4 \% \\
\text { (CDC } \\
\text { classification) } \\
\text { and } 1.0 \% \\
\text { (IOTF criteria) }\end{array}$ & $\begin{array}{l}\text { (IOTF criteria) } \\
\text { Girls = 5.3\% } \\
\text { (CDC } \\
\text { classification) } \\
\text { and } 0.6 \% \\
\text { (IOTF criteria) }\end{array}$ & \\
\hline 15 & $\begin{array}{l}\text { Munthali et al., } \\
2016 \text { [136] }\end{array}$ & Longitudinal & South Africa & Urban & 1824 & $5-18$ & $\begin{array}{l}\text { Male, } \\
\text { female }\end{array}$ & Prevalence & $\begin{array}{l}\text { Girls = late } \\
\text { onset } \\
\text { overweight } \\
(15 \%) \\
\text { Boys = early } \\
\text { onset } \\
\text { overweight } \\
\text { to normal } \\
(6 \%)\end{array}$ & $\begin{array}{l}\text { Girls = early } \\
\text { onset obesity } \\
\text { to } \\
\text { overweight } \\
(4.8 \%) \\
\text { Boys = early } \\
\text { onset } \\
\text { overweight } \\
\text { to obese } \\
(1.3 \%)\end{array}$ & IOTF \\
\hline 16 & $\begin{array}{l}\text { Negash et al., } \\
2017 \text { [68] }\end{array}$ & $\begin{array}{l}\text { Cross- } \\
\text { sectional }\end{array}$ & South Africa & Urban & 1559 & $7-18$ & $\begin{array}{l}\text { Male, } \\
\text { female }\end{array}$ & Prevalence & $22.9 \%$ & & IOTF \\
\hline 17 & $\begin{array}{l}\text { Ngwenya } \\
\text { et al., } 2017 \\
\text { [67] }\end{array}$ & $\begin{array}{l}\text { Cross- } \\
\text { sectional }\end{array}$ & South Africa & Urban & 175 & $13-19$ & $\begin{array}{l}\text { Male, } \\
\text { female }\end{array}$ & Prevalence & $15.4 \%$ & $8.6 \%$ & $\mathrm{BMI}$ \\
\hline 18 & $\begin{array}{l}\text { Pienaar, } 2015 \\
{[15]}\end{array}$ & Longitudinal & South Africa & $\begin{array}{l}\text { Rural, } \\
\text { urban }\end{array}$ & 574 & $6-9$ & $\begin{array}{l}\text { Male, } \\
\text { female }\end{array}$ & $\begin{array}{l}\text { Prevalence, } \\
\text { Trend }\end{array}$ & $\begin{array}{l}\text { Did not } \\
\text { report this }\end{array}$ & $16.7 \%$ & IOTF \\
\hline 19 & $\begin{array}{l}\text { Pretorius et al., } \\
2019 \text { [69] }\end{array}$ & $\begin{array}{l}\text { Cross- } \\
\text { sectional }\end{array}$ & South Africa & $\begin{array}{l}\text { Rural, } \\
\text { urban }\end{array}$ & 1785 & $6-12$ & $\begin{array}{l}\text { Male, } \\
\text { female }\end{array}$ & Prevalence & $27.3 \%$ & & $\mathrm{WHO}$ \\
\hline 20 & $\begin{array}{l}\text { Reddy et al., } \\
2012 \text { [113] }\end{array}$ & $\begin{array}{l}\text { Cross- } \\
\text { sectional }\end{array}$ & South Africa & $\begin{array}{l}\text { Rural, } \\
\text { urban }\end{array}$ & 4010 & $\begin{array}{l}\text { Mean = } \\
16.5\end{array}$ & $\begin{array}{l}\text { Male, } \\
\text { female }\end{array}$ & $\begin{array}{l}\text { Prevalence, } \\
\text { Trend }\end{array}$ & $\begin{array}{l}\text { Males = rates } \\
\text { increased } \\
\text { from 6.3\% in } \\
2002 \text { to } \\
11.0 \% \text { in } \\
2008 . \\
\text { Females = } \\
\text { rates } \\
\text { increased } \\
\text { from } 24.3 \% \\
\text { in 2002 to } \\
29.0 \% \text { in } \\
2008\end{array}$ & $\begin{array}{l}\text { Males = rates } \\
\text { doubled } \\
1.6 \% \text { in } 2002 \\
\text { to } 3.3 \% \text { in } \\
2008 \\
\text { Females = } \\
\text { rose from } 5.0 \\
\text { to } 7.5 \%\end{array}$ & IOTF \\
\hline 21 & $\begin{array}{l}\text { Sedibe et al., } \\
2018 \text { [118] }\end{array}$ & $\begin{array}{l}\text { Cross- } \\
\text { sectional }\end{array}$ & South Africa & $\begin{array}{l}\text { Rural, } \\
\text { urban }\end{array}$ & 3490 & $11-15$ & $\begin{array}{l}\text { Male, } \\
\text { female }\end{array}$ & Prevalence & \multicolumn{2}{|c|}{$\begin{array}{l}\text { More females overweight } \\
\text { and obese at both early and } \\
\text { mid-adolescents compared } \\
\text { to boys. Early adolescents =( } \\
\text { rural: } 17.34 \% \text { vs. } 9.52 \% \text {; } \\
\text { urban: } 36.15 \% \text { vs. } 27.89 \%) \text {, } \\
\text { and mid-adolescents = (rural } \\
22.33 \% \text { vs. } 5.50 \% \text {; urban: } \\
28.5 \% \text { vs. } 12.82 \%)\end{array}$} & WHO \\
\hline 22 & $\begin{array}{l}\text { Steyn et al., } \\
2011[129]\end{array}$ & $\begin{array}{l}\text { Secondary } \\
\text { analysis }\end{array}$ & South Africa & $\begin{array}{l}\text { Rural, } \\
\text { urban }\end{array}$ & 2469 & $1-9$ & $\begin{array}{l}\text { Male, } \\
\text { female }\end{array}$ & Prevalence & \multicolumn{2}{|c|}{$\begin{array}{l}24 \% \text { of children among } \\
\text { obese younger mothers }\end{array}$} & $\begin{array}{l}\text { WHO } \\
\text { IOTF }\end{array}$ \\
\hline 23 & $\begin{array}{l}\text { Symington } \\
\text { et al., } 2016 \\
{[130]}\end{array}$ & $\begin{array}{l}\text { Secondary } \\
\text { analysis }\end{array}$ & South Africa & $\begin{array}{l}\text { Rural, } \\
\text { urban }\end{array}$ & 519 & $3-9$ & $\begin{array}{l}\text { Male, } \\
\text { female }\end{array}$ & Prevalence & $12.0 \%$ & & $\begin{array}{l}\text { WHO } \\
\text { IOTF }\end{array}$ \\
\hline 24 & $\begin{array}{l}\text { Tathiah et al., } \\
2013 \text { [131] }\end{array}$ & $\begin{array}{l}\text { Secondary } \\
\text { analysis }\end{array}$ & South Africa & Rural & 963 & $7-14$ & Female & Prevalence & $9 \%$ & $3.8 \%$ & IOTF \\
\hline 25 & $\begin{array}{l}\text { Zeelie et al., } \\
2010 \text { [119] }\end{array}$ & $\begin{array}{l}\text { Cross- } \\
\text { sectional }\end{array}$ & South Africa & $\begin{array}{l}\text { Rural, } \\
\text { Urban }\end{array}$ & 232 & 5-19 & $\begin{array}{l}\text { Male, } \\
\text { female }\end{array}$ & Prevalence & \multicolumn{2}{|c|}{$\begin{array}{l}4.1 \% \text { of the boys and } 9.9 \% \text { of } \\
\text { the girls had a BMl above } \\
\text { the cut-off points }\end{array}$} & IOTF \\
\hline 26 & $\begin{array}{l}\text { Adegoke } \\
\text { et al., } 2009\end{array}$ & $\begin{array}{l}\text { Cross- } \\
\text { sectional }\end{array}$ & Nigeria & $\begin{array}{l}\text { Semi- } \\
\text { urban }\end{array}$ & 720 & $6-18$ & $\begin{array}{l}\text { Male, } \\
\text { female }\end{array}$ & Prevalence & $2.8 \%$ & $0.3 \%$ & IOTF \\
\hline
\end{tabular}


Table 2 Characteristics and findings of the included studies (Continued)

\begin{tabular}{|c|c|c|c|c|c|c|c|c|c|c|c|}
\hline & $\begin{array}{l}\text { Author \& } \\
\text { date }\end{array}$ & $\begin{array}{l}\text { Study } \\
\text { design }\end{array}$ & Country & Setting & $\begin{array}{l}\text { Sample } \\
\text { size }\end{array}$ & $\begin{array}{l}\text { Age } \\
\text { range } \\
\text { (years) }\end{array}$ & Gender & $\begin{array}{l}\text { Outcome } \\
\text { reported }\end{array}$ & $\begin{array}{l}\text { Prevalence } \\
\text { of } \\
\text { overweight }\end{array}$ & $\begin{array}{l}\text { Prevalence } \\
\text { of obesity }\end{array}$ & $\begin{array}{l}\text { Criteria for } \\
\text { assessment } \\
\text { of body } \\
\text { composition }\end{array}$ \\
\hline & [70] & & & & & & & & & & \\
\hline 27 & $\begin{array}{l}\text { Adesina et al., } \\
2012 \text { [76] }\end{array}$ & $\begin{array}{l}\text { Cross- } \\
\text { sectional }\end{array}$ & Nigeria & Urban & 960 & $10-19$ & $\begin{array}{l}\text { Male, } \\
\text { female }\end{array}$ & Prevalence & $6.3 \%$ & $1.8 \%$ & $\mathrm{BMI}$ \\
\hline 28 & $\begin{array}{l}\text { Akodu et al., } \\
2012 \text { [120] }\end{array}$ & $\begin{array}{l}\text { Cross- } \\
\text { sectional }\end{array}$ & Nigeria & Urban & 160 & $2-15$ & $\begin{array}{l}\text { Male, } \\
\text { female }\end{array}$ & Prevalence & $\begin{array}{l}\text { Did not } \\
\text { report on this }\end{array}$ & $\begin{array}{l}\text { Hemoglobin } \\
\text { genotype SS } \\
\text { subjects = } \\
2.5 \% \text {, and } \\
\text { hemoglobin } \\
\text { genotype AA } \\
\text { controls = } \\
3.8 \%\end{array}$ & WHO \\
\hline 29 & $\begin{array}{l}\text { Ene-Obong } \\
\text { et al., } 2012 \\
\text { [121] }\end{array}$ & $\begin{array}{l}\text { Cross- } \\
\text { sectional }\end{array}$ & Nigeria & Urban & 1599 & $5-18$ & $\begin{array}{l}\text { Male, } \\
\text { female }\end{array}$ & Prevalence & $11.4 \%$ & $2.8 \%$ & IOTF \\
\hline 30 & $\begin{array}{l}\text { Fetuga et al., } \\
2011 \text { [74] }\end{array}$ & $\begin{array}{l}\text { Cross- } \\
\text { sectional }\end{array}$ & Nigeria & $\begin{array}{l}\text { Semi- } \\
\text { urban }\end{array}$ & 1690 & $6-16$ & $\begin{array}{l}\text { Male, } \\
\text { female }\end{array}$ & Prevalence & $3.0 \%$ & $\begin{array}{l}\text { Did not } \\
\text { report on this }\end{array}$ & $\begin{array}{l}\text { CDC } \\
\text { WHO }\end{array}$ \\
\hline 31 & $\begin{array}{l}\text { Fetuga et al., } \\
2011 \text { [75] }\end{array}$ & $\begin{array}{l}\text { Cross- } \\
\text { sectional }\end{array}$ & Nigeria & $\begin{array}{l}\text { Semi- } \\
\text { urban }\end{array}$ & 1016 & $6-10$ & $\begin{array}{l}\text { Male, } \\
\text { female }\end{array}$ & Prevalence & $\begin{array}{l}\text { Did not } \\
\text { report on this }\end{array}$ & $0.5 \%$ & WHO \\
\hline 32 & $\begin{array}{l}\text { Maruf et al., } \\
2013 \text { [79] }\end{array}$ & $\begin{array}{l}\text { Cross- } \\
\text { sectional }\end{array}$ & Nigeria & Urban & 9014 & $2-18$ & $\begin{array}{l}\text { Male, } \\
\text { female }\end{array}$ & Prevalence & $6.1 \%$ & $0.8 \%$ & IOTF \\
\hline 33 & $\begin{array}{l}\text { Musa et al., } \\
2012 \text { [78] }\end{array}$ & $\begin{array}{l}\text { Cross- } \\
\text { sectional }\end{array}$ & Nigeria & $\begin{array}{l}\text { Rural, } \\
\text { urban }\end{array}$ & 3240 & $9-16$ & $\begin{array}{l}\text { Male, } \\
\text { female }\end{array}$ & Prevalence & $9.7 \%$ & $1.8 \%$ & IOTF \\
\hline 34 & $\begin{array}{l}\text { Nwaiwu et al., } \\
2015 \text { [122] }\end{array}$ & $\begin{array}{l}\text { Cross- } \\
\text { sectional }\end{array}$ & Nigeria & $\begin{array}{l}\text { Not } \\
\text { specified }\end{array}$ & 406 & $2-15$ & $\begin{array}{l}\text { Male, } \\
\text { female }\end{array}$ & Prevalence & $15.4 \%$ & $\begin{array}{l}\text { Did report } \\
\text { this }\end{array}$ & IOTF \\
\hline 35 & $\begin{array}{l}\text { Oduwole } \\
\text { et al., } 2012 \\
{[123]}\end{array}$ & $\begin{array}{l}\text { Cross- } \\
\text { sectional }\end{array}$ & Nigeria & Urban & 885 & $9-18$ & $\begin{array}{l}\text { Male, } \\
\text { female }\end{array}$ & Prevalence & $13.8 \%$ & $9.4 \%$ & $C D C$ \\
\hline 36 & $\begin{array}{l}\text { Okagua et al., } \\
2016 \text { [81] }\end{array}$ & $\begin{array}{l}\text { Cross- } \\
\text { sectional }\end{array}$ & Nigeria & Urban & 2282 & 10-19 & $\begin{array}{l}\text { Male, } \\
\text { female }\end{array}$ & Prevalence & $\begin{array}{l}14.6 \% \\
\text { (Females) } \\
11.4 \% \text { (Males) }\end{array}$ & $\begin{array}{l}5.2 \% \\
\text { (Females) } \\
3.8 \% \text { (Males) }\end{array}$ & WHO \\
\hline 37 & $\begin{array}{l}\text { Omisore et al., } \\
2015 \text { [80] }\end{array}$ & $\begin{array}{l}\text { Cross- } \\
\text { sectional }\end{array}$ & Nigeria & $\begin{array}{l}\text { Not } \\
\text { specified }\end{array}$ & 1000 & $10-19$ & $\begin{array}{l}\text { Male, } \\
\text { female }\end{array}$ & Prevalence & $\begin{array}{l}\text { 10.2\% (Males) } \\
5.3 \% \\
\text { (Females) }\end{array}$ & $\begin{array}{l}3.9 \% \text { (Males) } \\
2.0 \% \\
\text { (Females) }\end{array}$ & IOTF \\
\hline 38 & $\begin{array}{l}\text { Omuemu } \\
\text { et al., } 2010 \\
{[71]}\end{array}$ & $\begin{array}{l}\text { Cross- } \\
\text { sectional }\end{array}$ & Nigeria & Urban & 300 & $10-19$ & $\begin{array}{l}\text { Male, } \\
\text { female }\end{array}$ & Prevalence & $5.7 \%$ & & $C D C$ \\
\hline 39 & $\begin{array}{l}\text { Opara et al., } \\
2010 \text { [72] }\end{array}$ & $\begin{array}{l}\text { Cross- } \\
\text { sectional }\end{array}$ & Nigeria & $\begin{array}{l}\text { Rural, } \\
\text { urban }\end{array}$ & 985 & $2.5-14$ & $\begin{array}{l}\text { Male, } \\
\text { female }\end{array}$ & Prevalence & \multicolumn{2}{|c|}{$\begin{array}{l}11.1 \text { and } 0.2 \% \text { respectively in } \\
\text { private and public schools }\end{array}$} & WHO \\
\hline 40 & $\begin{array}{l}\text { Senbanjo } \\
\text { et al., } 2010 \\
{[73]}\end{array}$ & $\begin{array}{l}\text { Cross- } \\
\text { sectional }\end{array}$ & Nigeria & Urban & 570 & 5-19 & $\begin{array}{l}\text { Male, } \\
\text { female }\end{array}$ & Prevalence & $1.9 \%$ & & WHO \\
\hline 41 & $\begin{array}{l}\text { Senbanjo } \\
\text { et al., } 2011 \\
{[114]}\end{array}$ & $\begin{array}{l}\text { Cross- } \\
\text { sectional }\end{array}$ & Nigeria & Urban & 570 & 5-19 & $\begin{array}{l}\text { Male, } \\
\text { female }\end{array}$ & Trend & $\begin{array}{l}\text { Did not } \\
\text { report on this }\end{array}$ & $\begin{array}{l}5.0 \% \text { general } \\
\text { obesity }\end{array}$ & WHO \\
\hline 42 & $\begin{array}{l}\text { Senbanjo } \\
\text { et al., } 2012 \\
\text { [77] }\end{array}$ & $\begin{array}{l}\text { Cross- }^{-} \\
\text {sectional }\end{array}$ & Nigeria & Urban & 423 & $10-19$ & $\begin{array}{l}\text { Male, } \\
\text { female }\end{array}$ & Prevalence & $\begin{array}{l}\text { Did not } \\
\text { report on this }\end{array}$ & $\begin{array}{l}24.5 \% \text { central } \\
\text { obesity }\end{array}$ & WHO \\
\hline 43 & $\begin{array}{l}\text { Uwaezuoke } \\
\text { et al., } 2016 \\
\text { [124] }\end{array}$ & $\begin{array}{l}\text { Cross- }^{-} \\
\text {sectional }\end{array}$ & Nigeria & Urban & 2419 & 10-19 & $\begin{array}{l}\text { Male, } \\
\text { female }\end{array}$ & Prevalence & $\begin{array}{l}\text { Did not } \\
\text { report this }\end{array}$ & $\begin{array}{l}\text { Twelve of } 41 \\
\text { obese males } \\
\text { (29.3\%) and } \\
30 \text { of } 96 \\
\text { obese } \\
\text { females } \\
(31.3 \%)\end{array}$ & BMI \\
\hline 44 & Mekonnen & Cross- & Ethiopia & Rural, & 634 & $6-12$ & Male, & Prevalence & $8.8 \%$ & $3.1 \%$ & $\mathrm{WHO}$ \\
\hline
\end{tabular}


Table 2 Characteristics and findings of the included studies (Continued)

\begin{tabular}{|c|c|c|c|c|c|c|c|c|c|c|c|}
\hline & $\begin{array}{l}\text { Author \& } \\
\text { date }\end{array}$ & $\begin{array}{l}\text { Study } \\
\text { design }\end{array}$ & Country & Setting & $\begin{array}{l}\text { Sample } \\
\text { size }\end{array}$ & $\begin{array}{l}\text { Age } \\
\text { range } \\
\text { (years) }\end{array}$ & Gender & $\begin{array}{l}\text { Outcome } \\
\text { reported }\end{array}$ & $\begin{array}{l}\text { Prevalence } \\
\text { of } \\
\text { overweight }\end{array}$ & $\begin{array}{l}\text { Prevalence } \\
\text { of obesity }\end{array}$ & $\begin{array}{l}\text { Criteria for } \\
\text { assessment } \\
\text { of body } \\
\text { composition }\end{array}$ \\
\hline & $\begin{array}{l}\text { et al., } 2018 \\
\text { [82] }\end{array}$ & sectional & & urban & & & female & & & & \\
\hline 45 & $\begin{array}{l}\text { Moges et al., } \\
2018 \text { [83] }\end{array}$ & $\begin{array}{l}\text { Cross- } \\
\text { sectional }\end{array}$ & Ethiopia & Urban & 1276 & $10-19$ & $\begin{array}{l}\text { Male, } \\
\text { female }\end{array}$ & Prevalence & $17.0 \%$ & & WHO \\
\hline 46 & $\begin{array}{l}\text { Sorrie et al., } \\
2017 \text { [84] }\end{array}$ & $\begin{array}{l}\text { Cross- }^{-} \\
\text {sectional }\end{array}$ & Ethiopia & Urban & 504 & $3-5$ & $\begin{array}{l}\text { Male, } \\
\text { female }\end{array}$ & Prevalence & $13.8 \%$ & & WHO \\
\hline 47 & $\begin{array}{l}\text { Tadesse et al., } \\
2017 \text { [85] }\end{array}$ & $\begin{array}{l}\text { Cross- } \\
\text { sectional }\end{array}$ & Ethiopia & Urban & 462 & $3-6$ & $\begin{array}{l}\text { Male, } \\
\text { female }\end{array}$ & Prevalence & $6.9 \%$ & & WHO \\
\hline 48 & $\begin{array}{l}\text { Teshome } \\
\text { et al., } 2013 \\
\text { [87] }\end{array}$ & $\begin{array}{l}\text { Cross- } \\
\text { sectional }\end{array}$ & Ethiopia & Urban & 559 & $10-19$ & $\begin{array}{l}\text { Male, } \\
\text { female }\end{array}$ & Prevalence & $12.9 \%$ & $2.7 \%$ & WHO \\
\hline 49 & $\begin{array}{l}\text { Wakayo et al., } \\
2016 \text { [86] }\end{array}$ & $\begin{array}{l}\text { Cross- } \\
\text { sectional }\end{array}$ & Ethiopia & $\begin{array}{l}\text { Rural, } \\
\text { urban }\end{array}$ & 174 & $11-18$ & $\begin{array}{l}\text { Male, } \\
\text { female }\end{array}$ & Prevalence & $10.3 \%$ & & $\mathrm{WHO}$ \\
\hline 50 & $\begin{array}{l}\text { Pangani et al., } \\
2016 \text { [24] }\end{array}$ & $\begin{array}{l}\text { Cross- } \\
\text { sectional }\end{array}$ & Tanzania & Urban & 1781 & $8-13$ & $\begin{array}{l}\text { Male, } \\
\text { female }\end{array}$ & Prevalence & $15.9 \%$ & $6.7 \%$ & WHO \\
\hline 51 & $\begin{array}{l}\text { Mosha et al., } \\
2010 \text { [88] }\end{array}$ & $\begin{array}{l}\text { Cross- }^{-} \\
\text {sectional }\end{array}$ & Tanzania & Urban & 428 & $6-12$ & $\begin{array}{l}\text { Male, } \\
\text { female }\end{array}$ & Prevalence & $\begin{array}{l}\text { Did not } \\
\text { report on this }\end{array}$ & $\begin{array}{l}5.6 \% \text { in } \\
\text { Dodoma } \\
\text { compared to } \\
6.3 \% \text { in } \\
\text { Kinondoni } \\
\text { municipalities }\end{array}$ & BMl \\
\hline 52 & $\begin{array}{l}\text { Muhihi et al., } \\
2013 \text { [16] }\end{array}$ & $\begin{array}{l}\text { Cross- } \\
\text { sectional }\end{array}$ & Tanzania & $\begin{array}{l}\text { Rural, } \\
\text { urban }\end{array}$ & 446 & $6-17$ & $\begin{array}{l}\text { Male, } \\
\text { female }\end{array}$ & Prevalence & $\begin{array}{l}\text { Did not } \\
\text { report on this }\end{array}$ & $\begin{array}{l}\text { Overall, } 5.2 \% \\
(6.3 \% \text { in girls } \\
\text { and } 3.8 \% \text { in } \\
\text { boys) }\end{array}$ & IOTF \\
\hline 53 & $\begin{array}{l}\text { Mushengezi } \\
\text { et al., } 2014 \\
\text { [125] }\end{array}$ & $\begin{array}{l}\text { Cross- } \\
\text { sectional }\end{array}$ & Tanzania & Urban & 582 & $12-19$ & $\begin{array}{l}\text { Male, } \\
\text { female }\end{array}$ & Prevalence & $22.2 \%$ & & WHO \\
\hline 54 & $\begin{array}{l}\text { Mwaikambo } \\
\text { et al., } 2015 \\
\text { [89] }\end{array}$ & $\begin{array}{l}\text { Cross- } \\
\text { sectional }\end{array}$ & Tanzania & Urban & 1722 & $7-14$ & $\begin{array}{l}\text { Male, } \\
\text { female }\end{array}$ & Prevalence & $10.2 \%$ & $4.5 \%$ & IOTF \\
\hline 55 & $\begin{array}{l}\text { Adamo et al., } \\
2011 \text { [62] }\end{array}$ & $\begin{array}{l}\text { Cross- } \\
\text { sectional }\end{array}$ & Kenya & $\begin{array}{l}\text { Rural, } \\
\text { urban }\end{array}$ & 179 & $9-13$ & $\begin{array}{l}\text { Male, } \\
\text { female }\end{array}$ & Prevalence & \multicolumn{2}{|c|}{$\begin{array}{l}6.8 \% \text { of boys and } 16.7 \% \text { of } \\
\text { girls in urban Kenya }\end{array}$} & BMI \\
\hline 56 & $\begin{array}{l}\text { Gewa, } 2010 \\
{[137]}\end{array}$ & DHS & Kenya & $\begin{array}{l}\text { Rural, } \\
\text { urban }\end{array}$ & 1495 & $3-5$ & $\begin{array}{l}\text { Male, } \\
\text { female }\end{array}$ & Prevalence & $18.0 \%$ & $4.0 \%$ & WHO \\
\hline 57 & $\begin{array}{l}\text { Kimani- } \\
\text { Murage et al., } \\
2015 \text { [90] }\end{array}$ & $\begin{array}{l}\text { Cross- } \\
\text { sectional }\end{array}$ & Kenya & Urban & 3335 & $\begin{array}{l}\text { Under } \\
5\end{array}$ & $\begin{array}{l}\text { Male, } \\
\text { female }\end{array}$ & Prevalence & $8.8 \%$ & & WHO \\
\hline 58 & $\begin{array}{l}\text { Wachira et al., } \\
2018 \text { [91] }\end{array}$ & $\begin{array}{l}\text { Cross- } \\
\text { sectional }\end{array}$ & Kenya & Urban & 563 & $9-11$ & $\begin{array}{l}\text { Male, } \\
\text { female }\end{array}$ & Prevalence & $20.8 \%$ & & WHO \\
\hline 59 & $\begin{array}{l}\text { Choukem } \\
\text { et al., } 2017 \\
\text { [93] }\end{array}$ & $\begin{array}{l}\text { Cross- } \\
\text { sectional }\end{array}$ & Cameroon & Urban & 1343 & $3-13$ & $\begin{array}{l}\text { Male, } \\
\text { female }\end{array}$ & Prevalence & $\begin{array}{l}12.5 \%(13.2 \% \text { in } \\
11.8 \% \text { in boys) }\end{array}$ & $\mathrm{n}$ girls and & WHO \\
\hline 60 & $\begin{array}{l}\text { Navti et al., } \\
2014 \text { [94] }\end{array}$ & $\begin{array}{l}\text { Cross- } \\
\text { sectional }\end{array}$ & Cameroon & $\begin{array}{l}\text { Rural, } \\
\text { urban }\end{array}$ & 557 & $5-12$ & $\begin{array}{l}\text { Male, } \\
\text { female }\end{array}$ & Prevalence & $\begin{array}{l}17.0 \text { and } 17.8 \% \\
\text { boys respective }\end{array}$ & $\begin{array}{l}6 \text { in girls and } \\
\text { ely }\end{array}$ & WHO \\
\hline 61 & $\begin{array}{l}\text { Tchoubi et al., } \\
2015 \text { [132] }\end{array}$ & $\begin{array}{l}\text { Secondary } \\
\text { analysis }\end{array}$ & Cameroon & $\begin{array}{l}\text { Rural, } \\
\text { urban }\end{array}$ & 4518 & $<5$ & $\begin{array}{l}\text { Male, } \\
\text { female }\end{array}$ & Prevalence & $8.0 \%$ & & WHO \\
\hline 62 & $\begin{array}{l}\text { Wamba et al., } \\
2013 \text { [92] }\end{array}$ & $\begin{array}{l}\text { Cross- } \\
\text { sectional }\end{array}$ & Cameroon & Urban & 2689 & $8-15$ & $\begin{array}{l}\text { Male, } \\
\text { female }\end{array}$ & Prevalence & $\begin{array}{l}\text { Ranged from } \\
6.4 \text { to } 8.2 \% \text { in } \\
\text { boys and } \\
\text { from } 10.7 \text { to } \\
17.2 \% \text { in girls }\end{array}$ & $\begin{array}{l}\text { Ranged from } \\
1.4 \text { to } 5.5 \% \text { in } \\
\text { boys and } \\
\text { from } 2.4 \text { to } \\
8.6 \% \text { in girls }\end{array}$ & $\begin{array}{l}\text { IOTF } \\
\text { WHO } \\
\text { CDC } \\
\text { BMI database }\end{array}$ \\
\hline 63 & $\begin{array}{l}\text { Adom et al., } \\
2019 \text { [96] }\end{array}$ & $\begin{array}{l}\text { Cross- } \\
\text { sectional }\end{array}$ & Ghana & Urban & 543 & $8-11$ & $\begin{array}{l}\text { Male, } \\
\text { female }\end{array}$ & Prevalence & $16.4 \%$ & & WHO \\
\hline
\end{tabular}


Table 2 Characteristics and findings of the included studies (Continued)

\begin{tabular}{|c|c|c|c|c|c|c|c|c|c|c|c|}
\hline & $\begin{array}{l}\text { Author \& } \\
\text { date }\end{array}$ & $\begin{array}{l}\text { Study } \\
\text { design }\end{array}$ & Country & Setting & $\begin{array}{l}\text { Sample } \\
\text { size }\end{array}$ & $\begin{array}{l}\text { Age } \\
\text { range } \\
\text { (years) }\end{array}$ & Gender & $\begin{array}{l}\text { Outcome } \\
\text { reported }\end{array}$ & $\begin{array}{l}\text { Prevalence } \\
\text { of } \\
\text { overweight }\end{array}$ & $\begin{array}{l}\text { Prevalence } \\
\text { of obesity }\end{array}$ & $\begin{array}{l}\text { Criteria for } \\
\text { assessment } \\
\text { of body } \\
\text { composition }\end{array}$ \\
\hline 64 & $\begin{array}{l}\text { Kumah et al., } \\
2015 \text { [97] }\end{array}$ & $\begin{array}{l}\text { Cross- } \\
\text { sectional }\end{array}$ & Ghana & Urban & 500 & $10-20$ & $\begin{array}{l}\text { Male, } \\
\text { female }\end{array}$ & Prevalence & $12.2 \%$ & $0.8 \%$ & IOTF \\
\hline 65 & $\begin{array}{l}\text { Mohammed } \\
\text { et al., } 2012 \\
{[95]}\end{array}$ & $\begin{array}{l}\text { Cross- } \\
\text { sectional }\end{array}$ & Ghana & Urban & 270 & $5-15$ & $\begin{array}{l}\text { Male, } \\
\text { female }\end{array}$ & Prevalence & $\begin{array}{l}\text { Did not } \\
\text { report on this }\end{array}$ & $\begin{array}{l}10.9 \% \\
(\text { Girls }=15.0 \% \\
\text { Boys }=7.2 \%)\end{array}$ & WHO \\
\hline 66 & $\begin{array}{l}\text { Dos Santos } \\
\text { et al., } 2014 \\
\text { [98] }\end{array}$ & $\begin{array}{l}\text { Cross- } \\
\text { sectional }\end{array}$ & Mozambique & $\begin{array}{l}\text { Urban, } \\
\text { suburban }\end{array}$ & 3374 & $8-15$ & $\begin{array}{l}\text { Male, } \\
\text { female }\end{array}$ & $\begin{array}{l}\text { Prevalence, } \\
\text { Trend }\end{array}$ & $\begin{array}{l}5.0 \% \text { (Boys) } \\
11.2 \% \text { (Girls) }\end{array}$ & $\begin{array}{l}6.0 \% \text { (Boys) } \\
9.1 \% \text { (Girls) }\end{array}$ & WHO \\
\hline 67 & $\begin{array}{l}\text { Dos Santos } \\
\text { et al., } 2015 \\
\text { [99] }\end{array}$ & $\begin{array}{l}\text { Cross- } \\
\text { sectional }\end{array}$ & Mozambique & $\begin{array}{l}\text { Urban, } \\
\text { suburban }\end{array}$ & 323 & $10-15$ & $\begin{array}{l}\text { Male, } \\
\text { female }\end{array}$ & Prevalence & $\begin{array}{l}7.5 \% \text { (Boys) } \\
21.0 \% \text { (Girls) }\end{array}$ & & IOTF \\
\hline 68 & $\begin{array}{l}\text { Nagwa et al., } \\
2011[100]\end{array}$ & $\begin{array}{l}\text { Cross- } \\
\text { sectional }\end{array}$ & Sudan & Urban & 1138 & $10-18$ & $\begin{array}{l}\text { Male, } \\
\text { female }\end{array}$ & Prevalence & $10.8 \%$ & $9.7 \%$ & WHO \\
\hline 69 & $\begin{array}{l}\text { Salman et al., } \\
2011 \text { [101] }\end{array}$ & $\begin{array}{l}\text { Cross- } \\
\text { sectional }\end{array}$ & Sudan & Urban & 304 & $6-12$ & $\begin{array}{l}\text { Male, } \\
\text { female }\end{array}$ & Prevalence & $14.8 \%$ & $10.5 \%$ & CDC \\
\hline 70 & $\begin{array}{l}\text { Christoph } \\
\text { et al., } 2017 \\
{[102]}\end{array}$ & $\begin{array}{l}\text { Cross- } \\
\text { sectional }\end{array}$ & Uganda & $\begin{array}{l}\text { Rural, } \\
\text { urban }\end{array}$ & 148 & $11-16$ & $\begin{array}{l}\text { Male, } \\
\text { female }\end{array}$ & Prevalence & $1.4 \%$ & & WHO \\
\hline 71 & $\begin{array}{l}\text { Turi et al., } \\
2013 \text { [133] }\end{array}$ & $\begin{array}{l}\text { Secondary } \\
\text { analysis }\end{array}$ & Uganda & $\begin{array}{l}\text { Rural, } \\
\text { urban }\end{array}$ & 1099 & $<5$ & $\begin{array}{l}\text { Male, } \\
\text { female }\end{array}$ & Prevalence & $13.5 \%$ & & WHO \\
\hline 72 & $\begin{array}{l}\text { Wrotniak et al., } \\
2012 \text { [103] }\end{array}$ & $\begin{array}{l}\text { Cross- } \\
\text { sectional }\end{array}$ & Botswana & $\begin{array}{l}\text { Rural, } \\
\text { urban }\end{array}$ & 707 & $12-18$ & $\begin{array}{l}\text { Male, } \\
\text { female }\end{array}$ & Prevalence & $12.3 \%$ & $5.0 \%$ & WHO \\
\hline 73 & $\begin{array}{l}\text { Juwara et al., } \\
2016 \text { [104] }\end{array}$ & $\begin{array}{l}\text { Cross- } \\
\text { sectional }\end{array}$ & Gambia & Urban & 960 & $13-15$ & $\begin{array}{l}\text { Male, } \\
\text { female }\end{array}$ & Prevalence & \multicolumn{2}{|c|}{$\begin{array}{l}22.8 \% \text { in private schools and } \\
4.5 \% \text { in public schools }\end{array}$} & WHO \\
\hline 74 & $\begin{array}{l}\text { Van den Berg } \\
\text { et al., } 2014 \\
{[105]}\end{array}$ & $\begin{array}{l}\text { Cross- } \\
\text { sectional }\end{array}$ & Lesotho & Urban & 221 & 16 & $\begin{array}{l}\text { Male, } \\
\text { female }\end{array}$ & Prevalence & \multicolumn{2}{|c|}{$\begin{array}{l}8.3 \% \text { of boys and } 27.2 \% \text { of } \\
\text { girls }\end{array}$} & $\begin{array}{l}\text { WHO } \\
\text { CDC } \\
\text { IOTF }\end{array}$ \\
\hline 75 & $\begin{array}{l}\text { Caleyachetty } \\
\text { et al., } 2012 \\
{[106]}\end{array}$ & $\begin{array}{l}\text { Cross- } \\
\text { sectional }\end{array}$ & Mauritius & $\begin{array}{l}\text { Rural, } \\
\text { urban }\end{array}$ & 241 & $9-10$ & $\begin{array}{l}\text { Male, } \\
\text { female }\end{array}$ & Prevalence & $\begin{array}{l}15.8 \% \text { in } \\
\text { boys and } \\
18.9 \% \text { in girls }\end{array}$ & $\begin{array}{l}4.9 \% \text { in boys } \\
\text { and } 5.1 \% \text { in } \\
\text { girls }\end{array}$ & IOTF \\
\hline 76 & $\begin{array}{l}\text { Bovet et al., } \\
2010 \text { [107] }\end{array}$ & $\begin{array}{l}\text { Cross- } \\
\text { sectional }\end{array}$ & Seychelles & $\begin{array}{l}\text { Rural, } \\
\text { urban }\end{array}$ & 8462 & $\begin{array}{l}\text { Mean } \\
\text { ages; } \\
9.2,12.6 \\
\text { and } \\
15.3 \\
\text { years }\end{array}$ & $\begin{array}{l}\text { Male, } \\
\text { female }\end{array}$ & Prevalence & \multicolumn{2}{|c|}{$\begin{array}{l}37 \% \text { of boys in private } \\
\text { schools compared to } 15 \% \text { in } \\
\text { public schools } \\
33 \% \text { of girls in private } \\
\text { compared to } 20 \% \text { of those } \\
\text { in public schools }\end{array}$} & IOTF \\
\hline 77 & $\begin{array}{l}\text { Sagbo et al., } \\
2018 \text { [108] }\end{array}$ & $\begin{array}{l}\text { Cross- }^{-} \\
\text {sectional }\end{array}$ & Togo & Urban & 634 & $8-17$ & $\begin{array}{l}\text { Male, } \\
\text { female }\end{array}$ & Prevalence & $5.2 \%$ & $1.9 \%$ & IOTF \\
\hline 78 & $\begin{array}{l}\text { Kambondo } \\
\text { et al., } 2018 \\
{[109]}\end{array}$ & $\begin{array}{l}\text { Cross- } \\
\text { sectional }\end{array}$ & Zimbabwe & $\begin{array}{l}\text { Rural, } \\
\text { urban }\end{array}$ & 974 & $6-12$ & $\begin{array}{l}\text { Male, } \\
\text { female }\end{array}$ & Prevalence & $\begin{array}{l}\text { Did not } \\
\text { report on this }\end{array}$ & $\begin{array}{l}13.8 \% \text { in } \\
\text { urban } \\
\text { compared to } \\
2.3 \% \text { in rural } \\
\text { areas }\end{array}$ & IOTF \\
\hline 79 & $\begin{array}{l}\text { Muthuri et al., } \\
2016 \text { [110] }\end{array}$ & $\begin{array}{l}\text { Cross- }^{-} \\
\text {sectional }\end{array}$ & $\begin{array}{l}\text { Kenya, South } \\
\text { Africa }\end{array}$ & $\begin{array}{l}\text { Rural, } \\
\text { urban }\end{array}$ & 4725 & $9-11$ & $\begin{array}{l}\text { Male, } \\
\text { female }\end{array}$ & Prevalence & $\begin{array}{l}18.8 \text { and } \\
30.6 \% \text { in } \\
\text { Kenya and } \\
\text { South Africa } \\
\text { respectively }\end{array}$ & $\begin{array}{l}\text { Did not } \\
\text { report on this }\end{array}$ & WHO \\
\hline 80 & $\begin{array}{l}\text { Peltzer et al., } \\
2011 \text { [128] }\end{array}$ & $\begin{array}{l}\text { Secondary } \\
\text { analysis }\end{array}$ & $\begin{array}{l}\text { Ghana, } \\
\text { Uganda }\end{array}$ & $\begin{array}{l}\text { Not } \\
\text { specified }\end{array}$ & 5613 & $13-15$ & $\begin{array}{l}\text { Male, } \\
\text { female }\end{array}$ & Prevalence & $\begin{array}{l}10.4 \% \text { (Girls) } \\
\text { and 3.2\% } \\
\text { (Boys) }\end{array}$ & $\begin{array}{l}0.9 \% \text { (Girls) } \\
\text { and } 0.5 \% \\
\text { (Boys) }\end{array}$ & IOTF \\
\hline 81 & $\begin{array}{l}\text { Manyanga } \\
\text { et al., } 2014 \\
{[111]}\end{array}$ & $\begin{array}{l}\text { Cross- } \\
\text { sectional }\end{array}$ & $\begin{array}{l}\text { Benin, } \\
\text { Ghana, } \\
\text { Mauritania } \\
\text { and Malawi }\end{array}$ & $\begin{array}{l}\text { Not } \\
\text { specified }\end{array}$ & 23,496 & $11-17$ & $\begin{array}{l}\text { Male, } \\
\text { female }\end{array}$ & Prevalence & \multicolumn{2}{|c|}{$\begin{array}{l}8.7 \% \text { in Ghana, } 10.0 \% \text { in } \\
\text { Malawi, } 11.2 \% \text { in Benin, and } \\
24.3 \% \text { in Mauritania }\end{array}$} & WHO \\
\hline
\end{tabular}


in private and public schools in Uyo [72], and Senbanjo et al. reported $1.9 \%$ prevalence of overweight/obesity in Abeokuta [73]. Fetuga et al. cross-sectional surveys involving school children aged 6 to10 years in the semi-urban town of Sagamu, Ogun State reported 3.0\% overweight and $0.5 \%$ obesity using the WHO reference $[74,75]$. In their respective urban studies in 2012 among adolescents 10 to 19 years, Adesina et al. found overweight and obesity prevalence of 6.3 and 1.8\% in Port Harcourt; while in Abeokuta, Senbanjo et al. observed a $5 \%$ prevalence of general obesity and $24.5 \%$ central obesity [76, 77]. Similarly, Musa et al. reported overweight and obesity prevalence of 9.7 and 1.8\% respectively among adolescents in Benue State [78]. Maruf et al. reported an overall prevalence of $6.1 \%$ overweight and $0.8 \%$ obesity respectively in their cross-sectional study of Nigerian school children and adolescents aged 2 to 18 years [79]. Omisore et al. observed a significantly higher proportion of overweight and obesity in females (10.2 and 3.9\%) than males (5.3 and 2.0\%) in their 2015 study of 1000 adolescents aged 10 to 19 years in Osun State [80]. Okagua et al. study in Port Harcourt also reported a higher prevalence of overweight and obesity in females (14.6 and 5.2\%) than males (11.4 and 3.8\%) among adolescents aged 10 to 19 years [81].

In Ethiopia, all six included studies were crosssectional surveys that reported evidence on childhood obesity/overweight prevalence. Mekonnen et al. study in Bahir Dar City among 634 school children aged 6 to 12 years reported an overall overweight/obesity prevalence of $11.9 \%$ ( $8.8 \%$ overweight and $3.1 \%$ obesity) [82]; while Moges et al. reported the prevalence of overweight/obesity to be $17.0 \%$ among 1276 adolescents aged 10 to 19 years in Addis Ababa [83]. Sorrie et al. and Tadesse et al. identified a combined overweight/obesity prevalence of $13.8 \%$ in Gondar City and 6.9\% in Bahir Dar City respectively in their studies involving pre-school children [84, 85]. Wakayo et al. study in central Ethiopia reported an overweight and/or obesity prevalence of $10.3 \%$ among schooling adolescents aged 11 to 18 years old [86]. Teshome et al. also found $12.9 \%$ overweight and $2.7 \%$ obesity prevalence in their study involving 559 high school adolescents aged 10 to 19 years in the urban city of Hawassa [87].

In Tanzania, a comparative cross-sectional study by Mosha et al. in 2010 found that the prevalence of obesity among children 6 to 9 years was 5.6\% in Dodoma compared to $6.3 \%$ in Kinondoni municipalities; and for those aged 10 to 12 years, the prevalence of obesity was $3.9 \%$ compared to $5.8 \%$ in Dodoma and Kinondoni municipalities respectively [88]. Muhuhi et al. study in Dar es Salaam [16] reported a $5.2 \%$ overall prevalence of obesity (6.3\% in girls and $3.8 \%$ in boys). Mwaikambo et al. also in Dar es Salaam reported $10.2 \%$ overweight and $4.5 \%$ obesity rates among 1722 children aged 7 to 14 years [89].
Pangani et al. also studied 1781 primary school children aged 8-13 years and identified prevalence of overweight and obesity to be 15.9 and $6.7 \%$ respectively [24].

Four studies each from Kenya and Cameroon reported evidence of childhood obesity and/or overweight prevalence. Using data collected in the 2003 nationwide DHS in Kenya, Gewa et al. in 2009 reported approximately $18.0 \%$ overweight and $4.0 \%$ obesity prevalence from a random sample of 1495 pre-school children aged 3 to 5 years [137]. Adamo et al. study compared rural and urban Kenyan children ( $n=179,9-13$ years) and observed that whereas none of the rural Kenyan children were neither overweight nor obese, $6.8 \%$ of boys and $16.7 \%$ of girls in urban Kenya were found to be either overweight or obese [62]. In an urban poor setting of Nairobi, Kimani-Murage et al. [90] reported $8.8 \%$ overweight/obesity prevalence among a cohort of 3335 children under five. Wachira et al. examined 563 children aged 9 to 11 years attending 29 non-boarding primary schools in Nairobi, as part of the International Study of Childhood Obesity, Lifestyle and the Environment (ISCOLE) and found that $20.8 \%$ of participants were either overweight or obese [91]. In the urban city of Douala, Cameroon, Wamba and colleagues employed different international references and reported significant differences among the methods with a prevalence of overweight ranging from 6.4 to $8.2 \%$ in boys and from 10.7 to $17.2 \%$ in girls; whereas the prevalence of obesity ranged from 1.4 to $5.5 \%$ in boys and from 2.4 to $8.6 \%$ in girls [92]. A similar study in Douala by Choukem et al. utilized WHO BMI-for-age reference curves and observed that prevalence of obesity/overweight was $12.5 \%$ (13.2\% in girls and $11.8 \%$ in boys) [93]. Navti and colleagues studied 557 school children aged 5 to 12 years from both rural and urban areas of northwest Cameroon and found the prevalence of overweight/obesity to be 17.0 and $17.8 \%$ in girls and boys respectively [94]. The prevalence of obesity/overweight was $8.0 \%$ among 4518 children under 5 years in rural and urban Cameroon as reported by Tchoubi et al. [132].

Studies from three urban areas in Ghana were included in this study. In the city of Accra, Mohammed and Vuvor reported obesity prevalence of $10.9 \%$ (girls $=$ $15.0 \%$, boys $=7.2 \%$ ) among 270 basic school children between 5 and 15 years [95]; while Adom and colleagues reported an overall overweight/obesity prevalence of $16.4 \%$ among school children aged 8 to11 years in Aden$\tan$ municipality in 2019 [96]. Kumah et al. in 2015 also reported an overweight and obesity prevalence of 12.2 and $0.8 \%$ respectively from a sample of 500 high school students in Kumasi [97].

Two studies each were conducted in Mozambique, Sudan, and Uganda. A study by Dos Santos and colleagues in Maputo reported the prevalence of overweight/obesity 
to be $5.0 \% / 6.0 \%$ in boys and $11.2 \% / 9.1 \%$ in girls [98]. Dos Santos et al. study also in Maputo among 323 adolescents aged 10 to 15 years reported the prevalence of overweight/ obesity to be $7.5 \%$ in boys and $21.0 \%$ in girls [99]. In the Khartoum State of Sudan, Nagwa et al. observed that, the prevalence of overweight and obesity were 10.8 and $9.7 \%$ in adolescents 10 to 18 years old [100]; while Salman et al. found $14.8 \%$ overweight and $10.5 \%$ obesity among children between 6 and 12 years [101]. In Uganda, Turi et al. analyzed data from the 2011 DHS and found that $13.5 \%$ of children under 5 years were either overweight or obese [133]. Christoph et al. piloted a survey measuring weightrelated factors in 148 (11-16 years) rural and urban school children in Uganda and recorded an overweight and obesity prevalence rate of $1.4 \%$ [102].

Of the 81 studies included in this review, seven countries, Botswana, Gambia, Lesotho, Mauritius, Seychelles, Togo, and Zimbabwe reported one study each providing evidence on childhood overweight/obesity. In a crosssectional study, Wrotniak et al. identified overweight and obesity prevalence of 12.3 and $5.0 \%$ respectively in Botswana [103]. Juwara et al. recorded an overall overweight/obesity prevalence of $22.8 \%$ in private schools and $4.5 \%$ in public schools among adolescents in urban Gambia [104]. Among 225 16-year olds in urban Maseru, Lesotho, van den Berg et al. reported that 8.3\% of boys and $27.2 \%$ of girls were overweight and/or obese [105]. In Mauritius, Caleyachetty et al. found the prevalence of overweight to be $15.8 \%$ in boys and $18.9 \%$ in girls while obesity was $4.9 \%$ in boys and $5.1 \%$ in girls [106]. Bovet et al. study in Seychelles reported that 37\% of boys in private schools compared to $15 \%$ in public schools were overweight/obesity, whereas $33 \%$ of girls in private compared to $20 \%$ of those in public schools were either overweight or obese [107]. Sagbo et al. observed 5.2 and $1.9 \%$ prevalence of overweight and obesity respectively in urban areas of Lomé [108]. In Zimbabwe, Kambondo and colleagues studied 974 children aged 6 to 12 years and reported an obesity prevalence of $13.8 \%$ in urban compared to $2.3 \%$ in rural areas [109].

Three out of the 81 included studies were from multicountry settings. Muthuri et al. utilized data from a 12country study (ISCOLE) which included two SSA countries and reported an overweight prevalence of 18.8 and $30.6 \%$ in Kenya and South Africa respectively [110]. Peltzer and colleague performed a secondary analysis of existing data from the Global School-Based Health Survey (GSHS) from two SSA countries (Ghana and Uganda) in which they recorded prevalence of overweight/obesity of $10.4 \%$ in girls and $3.2 \%$ in boys, with 0.9 and $0.5 \%$ obesity among girls and boys, respectively [128]. Moreover, Mayanga et al. also analyzed data from 7 countries involved in the GSHS, out of which 4 were in SSA and reported a combined overweight/obesity prevalence $8.7 \%$ in Ghana, $10.0 \%$ in Malawi, $11.2 \%$ in Benin, and 24.3\% in Mauritania [111].

\section{Incidence of childhood overweight/obesity}

Of the 81 included studies only one study presented evidence on the incidence of childhood overweight and obesity. Lundeen et al. study in South Africa aimed to describe the gender differences in overweight and obesity from infancy to late adolescence among a cohort showed that the incidence of obesity was highest from 4 to 8 years to11-12 years in boys (6.8 cases per 1000 person-years) and from 11 to 12 years to $13-15$ years in girls (11.2 cases per 1000 person-years) [127]. This finding suggests limited studies focusing on the incidence of childhood obesity in SSA.

\section{Trends of childhood overweight/obesity}

Eight of the included studies reported evidence of childhood obesity and overweight trends in SSA. Of these, 6 (75\%) were conducted in South Africa, and one each (12.5\%) in Nigeria and Mozambique. In South Africa, Armstrong et al. compared data across two time periods (1994 and 2001/2004) and observed increasing secular trends of overweight and obesity from 1.2 to $13.0 \%$ and 0.2 to $3.3 \%$ among young South Africans over the 7-10 years period [63]. Feeley et al. collected anthropometric data from a birth cohort of 1298 children in the Soweto at age 13, 15, and 17 years and found that the combined overweight and obesity at 17 years was respectively 8.1 and $27 \%$ among males and females indicating a decrease in males and an increase in females from when they were 13 years old, though it was not stated by what magnitudes [134]. Kruger et al. study reported that the combined prevalence of overweight/obesity based on BMI cut-offs decreased significantly from $17.1 \%$ in 1999 to $14.0 \%$ in 2005 [112]. Lundeen and colleagues reported that obesity and overweight prevalence declined from age 1-2 years to 16-18 years among boys whereas among girls overweight and obesity prevalence increased throughout childhood (from 4 to 6 years to 16-18 years) among a cohort of 1172 children [127]. Pienaar et al. investigated changes in overweight and obesity prevalence among 574 children aged 6 to 9 years and found that obesity increased over the 3 -years by $4 \%$ from $12.5 \%$ at baseline to $16.7 \%$ during follow-up [15]. This change was higher in whites $(4.2 \%)$ than blacks $(2.0 \%)$ and in boys (3.2\%) compared to girls (2.4\%). Reddy et al. [113] also observed that overweight rates increased from $6.3 \%$ in 2002 to $11.0 \%$ in 2008 among male adolescents and from $24.3 \%$ in 2002 to $29.0 \%$ in 2008 among female adolescents; while obesity rates more than doubled among male adolescents from $1.6 \%$ in 2002 to $3.3 \%$ in 2008 compared to a rise from 5.0 to $7.5 \%$ among females who participated in the South African National Youth Risk 
Behaviour Survey in 2002 and 2008. Senbanjo et al. study aimed to determine current nutritional status and its changes between 1983 and 2006 among school children and adolescents in Abeokuta, Nigeria found that obesity prevalence rose from 1.7 to $3.3 \%$ in males and from 2.6 to $5.1 \%$ in females over the period [114]. In Mozambique, Dos Santos et al. examined secular trends in the nutritional status of children and adolescents for 1992, 1999, and 2012 and reported that obesity increased from 0.8 to 1.6 and 6.0 respectively in boys and from 1.8 to 4.5 and 9.1 respectively in girls [98]. This finding also suggests limited studies focusing on trends of childhood overweight obesity in SSA.

\section{Discussion}

This scoping review presented evidence on the prevalence, incidence, and trends of childhood obesity in SSA from studies published between January 2009 and June 2019. The review showed that a total of 81 studies were published within the period from 17 SSA countries including 3 multi-country studies. Most (53\%) of the studies were conducted in South Africa (31\%) and Nigeria (22\%) with 11 countries reporting less than 5 studies each. Moreover, the majority $(81.5 \%)$ of the studies were cross-sectional and most studies (79) focused on both male and female participants with no study reporting on only male participants. The review further revealed that almost all the included studies (80/81) reported about prevalence with only 1 study reporting about incidence while 8 reported on trends. This study's findings suggest limited evidence on trends and incidence of childhood obesity in SSA.

We found 81 studies presenting evidence on the burden of childhood obesity published in 20 SSA countries between 2009 and July 2019. This points to a lack of evidence from over $60 \%$ of the countries included in the WHO list of SSA countries [140, 141], which presents huge literature gaps. This supports findings from the systematic review by Keino et al. which explored the determinants of stunting and overweight in SSA and reported a paucity of literature from most parts of SSA [49]. It further backs findings from Jaacks et al. systematic review of current evidence on maternal and child overweight and obesity in the context of undernutrition which indicated limited scientific literature, especially in LMIC to support such reviews [54].

The highest number of studies were reported in South Africa (31\%) which shows a high level of interest in childhood obesity research in that country. This interest may stem from the fact that several studies have reported that South Africa leads on the league table of countries with the highest prevalence of obesity in Africa [27, 142]. The WHO Global Status Report on NCDs, 2010 indicates that the prevalence of overweight is highest in upper-middle-income countries (UMICs) while the fastest rise in overweight is in lower-middle-income countries [143]. Agreeably, most of the included studies in this review, $74.1 \%(60 / 81)$ were from either UMIC whilst 21 and $1.2 \%$ were from LMIC and low-income countries respectively with only one study (1.2\%) was from a high-income country (Seychelles) based on the 20192020 World Bank country classifications [144] (Table 3).

We further observed that majority of the included studies utilized either WHO growth references (48.2\%) or Cole et al. and IOTF criteria (34.6\%) with few employing BMI cut-off (7.6\%) and CDC classifications (3.7\%). This agrees with findings from systematic reviews by Monyeki et al. and Muthuri et al. which stated that most of the studies in their review employed different widely accepted (Cole et al. and IOTF, CDC and WHO) international cut-off points [20,51]. However, Monyeki et al. further stated that the Cole-IOTF and WHO 2006 growth standards tend to overestimate overweight and obesity prevalence hence making it difficult to interpret findings across studies due to the variations in these reference standards [51]. In contrast, Bentham et al. study aimed to determine worldwide trends in mean BMI in children and adolescents reported that prevalence using WHO criteria were higher than those of IOTF and CDC but yielded similar trends [5], and this tends to agree with findings from this study. We, therefore, recommend that future researches aim at comparing childhood overweight and obesity prevalence should be based on a single reference criterion to ensure uniformity and comparability of data.

Among children under 5 years, the combined prevalence of overweight and obesity reported ranged from a minimum of $8.0 \%$ in Cameroon [132] to a maximum of $16.0 \%$ in South Africa [126]. This confirms reports by UNICEF, WHO, and the World Bank which ranked Southern Africa as the region with the highest prevalence of overweight among children under 5 years (14.6\%), followed by Central Asia (11.6\%) and Northern Africa (11.0\%) [145]. This finding presents serious policy implications and the urgent need to implement interventions to reverse this trend and forestall future adverse consequences. Moreover, the highest reported prevalences among children and adolescents were in Seychelles, South Africa, Lesotho, and Mauritania. In contrast, Uganda, Cameroon, Tanzania, and Nigeria recorded some of the lowest prevalence. There was a higher prevalence of overweight/obesity among girls than boys in private than in public schools, and in urban than in rural areas in most of the study settings. This contrasts with findings by Duncan et al. in which the prevalence of overweight and obesity was higher among males than females in Brazil [146]. Similarly, Adamo et al. who compared children from rural and urban Kenya to their counterparts in Canada reported that 
Table 3 2019-2020 World Bank country income level classifications of the countries the included studies were conducted $(N=20)$

\begin{tabular}{|c|c|c|c|}
\hline High-income country & Upper-middle-income countries & Lower-middle-income countries & Low-income countries \\
\hline \multirow[t]{8}{*}{ Seychelles } & Botswana & Cameroon & Ethiopia \\
\hline & Mauritius & Ghana & Mozambique \\
\hline & South Africa & Kenya & Tanzania \\
\hline & & Lesotho & Togo \\
\hline & & Nigeria & Uganda \\
\hline & & Sudan & Malawi \\
\hline & & Zimbabwe & Benin \\
\hline & & Mauritania & The Gambia \\
\hline Total = 1 & Total $=3$ & Total $=8$ & Total $=8$ \\
\hline
\end{tabular}

while obesity/overweight was non-existent in the rural Kenyan population, urban Kenyan children were anthropometrically similar to their contemporaries in Canada [62]. This suggests a possible nutritional transitional in urban areas which may be partly explained by the adoption of more western and obesogenic lifestyles by urban dwellers in SSA. These findings again point to an obvious fact that obesity and overweight rates in the region are currently comparable and in some instances exceed rates in highly developed countries; where reported prevalence range from $10 \%$ in Denmark to $31 \%$ in the United State [147].

Our findings also revealed rapidly increasing trends of childhood overweight and obesity in SSA particularly among adolescent girls $[111,130,136]$. This supports reports by Tzioumis and colleagues that the global prevalence of obesity has increased in all regions of the world with developing countries recording greater absolute numbers of affected children and higher relative increases [148]. According to the WHO, while most countries in the world are experiencing a rapid upsurge in childhood obesity, this situation is even more alarming in LMICs, especially in SSA where the "dual burden" of infectious diseases and under-nutrition co-existing with high rates of NCDs risk factors such as obesity and overweight is prevalent $[6,140,141]$. $\mathrm{Ng}$ et al. further reported after their systematic analysis of the Global Burden of Disease study from 1980 to 2013 that, not only is obesity increasing but no national success stories have been reported in the past 33 years [142]. Lobstein and Jackson-Leach in 2016 estimated that by 2025 some 268 million children aged $5-17$ years globally may be overweight, including 91 million obese based on the assumption that no policy interventions prove effective at changing current trends [21]. They hence concluded that the WHOs Sustainable Development Goal (SDG) target to halt the rise in obesity by 2025 and reduce premature mortality due to NCDs by one third by the year 2030 is unlikely to be met and that health service providers will need to plan for a significant increase in obesity-related comorbidities. Bollyky et al. have indicated elsewhere that lower-income countries that face the most rapid surge in NCD burden are also the least prepared and are expected to make the least increases in health expenditure [143].

\section{Implications for research}

We observed a paucity of literature on the outcomes, especially on incidence and trends of childhood obesity and overweight from most countries in the SSA region. The studies also utilized different anthropometric methods of assessment which made it difficult for comparisons. Moreover, the studies which reported on trends were not very current. We, therefore, recommend that (i) more studies should be conducted especially on time trends and incidence of childhood obesity/overweight in different setting across SSA, (ii) future reviews should focus on studies utilizing one specific assessment criterion for easy interpretation and comparability of results, (iii) the finding of the study presents alarming high prevalence and rising trends of childhood obesity and overweight hence, we recommend more intervention studies that will develop practicable solutions to address this challenge, and (iv) we also recommend follow-up studies to map evidence on the risk factors of childhood obesity in SSA, in order to guide efforts aimed at preventing the problem.

\section{Implications for practice}

This study findings demonstrate an urgent need for a more concerted effort among governments in SSA and the global health community in tackling the rising burden of obesity/overweight and NCDs in the region. To this end, policymakers are encouraged to formulate policies that facilitate the identification, management, and most especially prevention of childhood overweight/ obesity. Governments in SSA should also endeavor to increase funds and resource allocation towards combating this problem which will potentially reduce the NCD burden in the region. Finally, policy interventions should be holistic, context-specific, age-appropriate, culturally and socially responsive, and multi-sectorial in nature to increase their chances of success. 


\section{Strengths and limitations of the study}

We followed all the steps required of systematic reviews in reporting this study except the registration in PROSPERO. The study protocol was however published in a peer-reviewed journal [31]. We also performed a thorough, systematic, and comprehensive search for literature on the prevalence, incidence, and trends of childhood obesity in SSA using MeSH terms to address alternative terminologies of the keywords. This study also had many limitations. This study included only published articles. This potentially excluded relevant information that may have been documented on national and international organizations registries or websites such as the WHO, ministries of health, government statistical service, and other grey literature. The language, and geographical setting limitations perhaps, exclude important evidence hence affecting the external generalization of the results. For example, four studies that were published in French but provided abstracts in English were able to pass the abstract screening stage but were dropped at full-text screening stage due to lack of expertise to translate the French language. This study also sought to provide recent evidence ( 10 years) hence, the date limitation from 2009 to 2019 probably excluded useful sources of evidence. As part of this study's published protocol [31], we planned to appraisal the methodological quality of the included studies, but this was not done due to the explorative nature of scoping reviews. Unlike systematic reviews and metaanalysis, quality appraisal is also not mandatory for a scoping review study. Moreover, the risk of bias might not be useful considering the number of included studies. Nonetheless, we hope to conduct a full systematic review and meta-analysis as a follow-up study. Hence, we will perform the methodological quality appraisal and additionally report the risk of bias in the next phase of this study.

\section{Conclusion}

Our study findings indicate increasing prevalence, incidence, and trends of childhood obesity in SSA. However, this study suggests limited studies focusing on childhood obesity/overweight in most SSA countries. The evidence demonstrated by this review should, therefore, serve as a wake-up call for researchers in SSA and the global health community to expedite action through proactive and pragmatic interventions to stem the rise of childhood obesity/overweight and consequent NCD burden in SSA. Finally, we reiterate the statement by the Pan American Health Organization that although the cost of NCDs is high, the cost of inaction is even higher and that paying for NCD prevention and control is much more than a cost, but rather, an investment for the future [140].

\section{Supplementary Information}

The online version contains supplementary material available at https://doi. org/10.1186/s13690-020-00491-2.

Additional file 1. The Preferred Reporting Items for Systematic reviews and Meta-Analyses extension for Scoping Reviews (PRISMA-SCR) Checklist.

Additional file 2. Electronic databases search results for title screening.

\begin{abstract}
Abbreviations
BMI: Body Mass Index; CDC: Centers for Disease Control and Prevention; DHS: Demographic and Health Survey; GBD: Global Burden of Disease; GSHS: Global School-Based Health Survey; IOTF: International Obesity Task Force; ISCOLE: International Study of Childhood Obesity, Lifestyle and Environment; LIC: Low-Income Country; LMIC: Lower-Middle-Income Country; MMAT: Mixed Methods Appraisal Tool; NCD: Non-Communicable Disease; NCHS: National Center for Health Statistics; PEO: Population, Exposure and Outcomes; PRISMA-ScR: Preferred Reporting Items for Systematic Reviews and Meta-Analyses; SDG: Sustainable Development Goal; SSA: Sub-Saharan Africa; UMIC: Upper-Middle-Income Country; WHO: World Health Organization
\end{abstract}

\section{Acknowledgments}

We are thankful to the Catholic University College of Ghana, Fiapre and the University of KwaZulu-Natal, Durban for providing us with essential resources to complete this review.

\section{Authors' contributions}

FID and DK conceptualized the study. FID wrote the manuscript. FID, MAM and MY contributed to the abstract and full-text screening. DK contributed to the full-text screening. D.K and VB critically reviewed the manuscript and made revisions. All the authors approved the final draft.

\section{Funding}

No funding was obtained for this study.

\section{Availability of data and materials}

The data supporting the conclusion of this paper are available through the detailed reference list. No original datasets are presented since this was a review of previously existing literature.

\section{Ethics approval and consent to participate}

This study is a systematic scoping review that relied solely on existing literature. Hence, ethical approval was not required since there were no human participants.

\section{Consent for publication}

Not applicable.

\section{Competing interests}

The authors declared no competing interests.

\section{Author details}

${ }^{1}$ Department of Public Health, Faculty of Health and Allied Sciences, Catholic University College of Ghana, Fiapre, Sunyani, Ghana. ${ }^{2}$ Research for Sustainable Development Consult, Sunyani, Ghana. ${ }^{3}$ Department of Public Health Medicine, School of Nursing and Public Health, University of KwaZulu-Natal, Durban 4001, South Africa.

Received: 12 June 2020 Accepted: 20 October 2020

Published online: 29 October 2020

\section{References}

1. Glasgow S, Schrecker T. Health \& Place the double burden of neoliberalism? Noncommunicable disease policies and the global political economy of risk. Health Place. 2016;39:204-11. Available from:. https://doi.org/10.1016/j. healthplace.2016.04.003.

2. Benziger CP, Roth GA, Moran AE. The global burden of disease study and the preventable burden of NCD. Glob Heart. 2016;11(4):393-7. Available from:. https://doi.org/10.1016/j.gheart.2016.10.024. 
3. Kengne AP, Bentham J, Zhou B, Peer N, Matsha TE, Bixby H, et al. Trends in obesity and diabetes across Africa from 1980 to 2014: an analysis of pooled population-based studies. Int J Epidemiol. 2017;46(5):1421-32 Available from: https://academic.oup.com/ije/article-abstract/46/5/1421/3861188. Cited 2019 May 20.

4. Mortality GBD, Collaborators D. Global, regional, and national age - sex specifi c all-cause and cause-specifi c mortality for 240 causes of death , 19902013 : a systematic analysis for the Global Burden of Disease Study 2013. Lancet. 2014;385(9963):117-71. https://doi.org/10.1016/S0140-6736(14)61682-2.

5. Bentham J, Di Cesare M, Bilano V, Bixby H, Zhou B, Stevens GA, et al. Worldwide trends in body-mass index, underweight, overweight, and obesity from 1975 to 2016: a pooled analysis of 2416 population-based measurement studies in 128.9 million children, adolescents, and adults. Lancet. 2017;390(10113):2627-42.

6. W.H.O. WHO Media centre Obesity and overweight Fact sheet; 2017. p. 3-7. Available from: http://www.who.int/mediacentre/factsheets/fs311/en/.

7. WHO. Commission on Ending Childhood Obesity: WHO; 2016. Available from: http://www.who.int/end-childhood-obesity/en/.

8. Frederick CB, Snellman K, Putnam RD. Increasing socioeconomic disparities in adolescent obesity. Proc Natl Acad Sci. 2014;111(4):1338-42 Available from: http://login.research4life.org/tacsgr1www_pnas_org/ content/111/4/1338.abstract. Cited 2019 Mar 18.

9. Apovian CM. Obesity: definition, comorbidities, causes, and burden. Am J Manag Care. 2016;22(7 Suppl):s176-85 Available from: http://www.ncbi.nlm. nih.gov/pubmed/27356115. Cited 2019 Mar 18.

10. Swinburn BA, Sacks G, Hall KD, McPherson K, Finegood DT, Moodie ML, et al. The global obesity pandemic: shaped by global drivers and local environments. Lancet 2011;378(9793):804-814. Available from: https://doi. org/10.1016/S0140-6736(11)60813-1

11. WHO. Obesity: WHO; 2014. Available from: https://www.who.int/topics/ obesity/en/. Cited 2019 Mar 19

12. Seidell JC, Halberstadt J. The global burden of obesity and the challenges of prevention. Ann Nutr Metab. 2015;66 Suppl 2(2):7-12 Available from: https://www.karger.com/Article/FullText/375143. Cited 2019 May 3.

13. Styne DM, Arslanian SA, Connor EL, Afrooqi IS, Murad MH, Silverstein JH, et al. Pediatric obesity-assessment, treatment, and prevention: An endocrine society clinical practice guideline. J Clin Endocrinol Metab. 2017;102(3):70957. Available from: http://www.embase.com/search/results?subaction= viewrecord\&from=export\&id=L614843766\%5Cn10.1210/jc.2016-2573\%5Cn. https://doi.org/10.1210/jc.2016-2573.

14. Percy R. Primary Care Management of Pediatric Obesity; 2017.

15. Pienaar AE. Prevalence of overweight and obesity among primary school children in a developing country: NW-CHILD longitudinal data of 6-9-yr-old children in South Africa. BMC Obes. 2015;2(1):2. https://doi.org/10.1186/ s40608-014-0030-4 Cited 2019 May $19 .$.

16. Muhihi AJ, Mpembeni RNM, Njelekela MA, Anaeli A, Chillo O, Kubhoja S, et al. Prevalence and determinants of obesity among primary school children in Dar es Salaam, Tanzania. Arch Public Heal. 2013;71(1):26. https:// doi.org/10.1186/0778-7367-71-26 Cited 2019 May 19.

17. Ezzati M, Alan D, Lopez AR, Murray CJL. Overweight and Obesity (Body Mass Index). Comp Quantif Heal Risks. 2004;1(1):497-6.

18. World Cancer Research Fund. Diet, nutrition, physical activity and colorectal cancer. 2018; Available from: https://www.wcrf.org/sites/default/files/ Colorectal-Cancer-2017-Report.pdf.

19. Wallander JL, Kerbawy S, Toomey S, Lowry R, Elliott MN, Escobar-Chaves SL, et al. Is obesity associated with reduced health-related quality of life in Latino, black and white children in the community? Int J Obes. 2013;37(7): 920-5. Available from:. https://doi.org/10.1038/ijo.2013.31.

20. Muthuri SK, Francis CE, Wachira L-JM, Leblanc AG, Sampson M, Onywera VO, et al. Evidence of an overweight/obesity transition among school-aged children and youth in Sub-Saharan Africa: a systematic review. PLoS One. 2014; 9(3):e92846. https://doi.org/10.1371/journal.pone.0092846 Cited 2019 May 19.

21. Lobstein T, Jackson-Leach R. Planning for the worst: estimates of obesity and comorbidities in school-age children in 2025. Pediatr Obes. 2016;11(5):321-5.

22. Onywera VO. Childhood obesity and physical inactivity threat in Africa: strategies for a healthy future. Glob Health Promot. 2010;17(2 Suppl):45-6. https://doi.org/10.1177/1757975910363937 Cited 2019 May 19.

23. Mozaffari $\mathrm{H}$, Nabaei B. Obesity and related risk factors. Indian J Pediatr. 2007; 74(3):265-7.

24. Pangani IN, Kiplamai FK, Kamau JW, Onywera VO. Prevalence of Overweight and Obesity among Primary School Children Aged 8-13 Years in Dar es
Salaam City, Tanzania. Adv Prev Med. 2016;2016:1-5 Available from: http:// www.hindawi.com/journals/apm/2016/1345017/. Cited 2019 May 19.

25. Kanter R, Caballero B. Global gender disparities in obesity: a review. Adv Nutr. 2012; 3(4):491-8 Available from: http://www.ncbi.nlm.nih.gov/pubmed/22797984\%0. http://www.pubmedcentral.nih.gov/articlerender.fcgi?. Cited 2019 May 20.

26. Gellner R, Domschke W. Epidemiologie der Adipositas. Chirurg. 2008;79(9): 807-18. https://doi.org/10.1007/s00104-008-1534-6 Cited 2019 May 20.

27. WHO. Non communicable Diseases Country Profiles 2014, vol. 2014. Genève: WHO Press; 2014. p. 1-210. Available from: http://www.who.int/ $\mathrm{nmh} /$ countries/ben_en.pdf?ua=1.

28. Arksey H, Malley LO. Scoping studies: Towards a methodological framework; 2005. p. 19-32.

29. Levac D, Colquhoun H, O'Brien KK. Scoping studies: Advancing the methodology. Implement Sci. 2010;5(1):69 Available from: http://www.ncbi. nlm.nih.gov/pubmed/20854677. Cited 2019 Mar 23.

30. The Joanna Briggs Institute. The Joanna Briggs Institute Reviewers' Manual 2015: Methodology for JBI scoping reviews: Joanne Briggs Inst; 2015. p. 1 24. Available from: http://joannabriggs.org/assets/docs/sumari/ ReviewersManual_Mixed-Methods-Review-Methods-2014-ch1.pdf.

31. Danquah Fl, Yeboah M, Bawuntuo V, Kuupiel D. Mapping evidence on the burden and distribution of childhood Obesity in sub- Saharan Africa: a systematic Scoping review protocol. BMC Nutr. 2019;8(1):274.

32. Moher D, Liberati A, Tetzlaff JAD. PRISMA 2009 Flow Diagram. PRISMA Statement. 2009;6:1000097.

33. Abrahams Z, McHiza Z, Steyn NP. Diet and mortality rates in Sub-Saharan Africa: Stages in the nutrition transition. BMC Public Health. 2011;11(1):801. https://doi.org/10.1186/1471-2458-11-801 Cited 2019 May 20.

34. Afrifa-Anane E, Agyemang C, SNA C, Ogedegbe G, De-Graft Aikins A. The association of physical activity, body mass index and the blood pressure levels among urban poor youth in Accra, Ghana. BMC Public Health. 2015; 15(1):269. https://doi.org/10.1186/s12889-015-1546-3 Cited 2019 May 20.

35. Chedjou-Nono E, Sap S, Choukem S-P, Ngosso Tetanye I, Nebongo D, Koki Ndombo O. Cardiometabolic profile of obese children in a sub-Saharan African setting: a cross-sectional study. BMC Pediatr. 2017;17(1):129. https:// doi.org/10.1186/s12887-017-0880-2 Cited 2019 May 19.

36. Craig E, Reilly JJ, Bland R. Risk factors for overweight and overfatness in rural South African children and adolescents; 2015. p. 1-10.

37. Humbwavali JB, Giugliani C, Silva ICM d, Duncan BB. Temporal trends in the nutritional status of women and children under five years of age in subSaharan African countries: ecological study. Sao Paulo Med J. 2018;136(5):45463 Available from: http://www.scielo.br/scielo.php?script=sci_arttext\&pid= S1516-31802018000500454\&lng=en\&tIng=en. Cited 2019 May 19.

38. Locks LM, Mwiru RS, Mtisi E, Manji KP, McDonald CM, Liu E, et al. Infant Nutritional Status and Markers of Environmental Enteric Dysfunction are Associated with Midchildhood Anthropometry and Blood Pressure in Tanzania. J Pediatr. 2017;187:225-233.e1 Available from: https://linkinghub. elsevier.com/retrieve/pii/S0022347617304882. Cited 2019 May 19

39. Njelekela M, Muhihi A, Mpembeni RM, Anaeli A, Chillo O, Kubhoja S, et al. Knowledge and attitudes towards obesity among primary school children in Dar es Salaam, Tanzania. Niger Med J. 2015;56(2):103 Available from: http:// www.nigeriamedj.com/text.asp?2015/56/2/103/150692. Cited 2019 May 20.

40. Muhihi AJ, Njelekela MA, Mpembeni RNM, Muhihi BG, Anaeli A, Chillo O et al. Elevated blood pressure among primary school children in Dar es salaam, Tanzania: Prevalence and risk factors. BMC Pediatr. 2018;18(1):54. https://doi.org/10.1186/s12887-018-1052-8 Cited 2019 May 19.

41. Munthali RJ, Sahibdeen V, Kagura J, Hendry LM, Norris SA, Ong KK, et al. Genetic risk score for adult body mass index associations with childhood and adolescent weight gain in an African population. Genes Nutr. 2018; 13(1):24. https://doi.org/10.1186/s12263-018-0613-7 Cited 2019 May 20.

42. Omigbodun $\mathrm{OO}$, Adediran Kl, Akinyemi JO, Omigbodun AO, Adedokun BO, Esan $\mathrm{O}$. Gender and rural-urban differences in the nutritional status of inschool adolescents in south-western Nigeria. J Biosoc Sci. 2010;42(5):653-76 Available from: https://www.cambridge.org/core/product/identifier/S0021 932010000234/type/journal_article. Cited 2019 May 19.

43. Onywera VO. Commentary Childhood obesity and physical inactivity threat in Africa: strategies for a healthy future. Glob Health Promot. 2010;2:45-6.

44. Pisa PT, Pedro TM, Kahn K, Tollman SM, Pettifor JM, Norris SA. Nutrient patterns and their association with socio-demographic, lifestyle factors and obesity risk in rural South African adolescents. Nutrients. 2015;7(5): 3464-82 Available from: http://www.mdpi.com/2072-6643/7/5/3464. Cited 2019 May 19 
45. Price AJ, Crampin AC, Amberbir A, Kayuni-Chihana N, Musicha C, Tafatatha $T$, et al. Prevalence of obesity, hypertension, and diabetes, and cascade of care in sub-Saharan Africa: a cross-sectional, population-based study in rural and urban Malawi. Lancet Diabetes Endocrinol. 2018;6(3):208-22. Available from:. https://doi.org/10.1016/S2213-8587(17)30432-1.

46. Senbanjo IO, Oshikoya KA, Olutekunbi OA, Njokanma OF. Body fat distribution of children and adolescents in Abeokuta, Southwest Nigeria. Am J Phys Anthropol. 2013;150(4):647-54. Available from:. https://doi.org/10. 1002/ajpa.22241.

47. Mpembeni RNM, Muhihi AJ, Maghembe M, Ngarashi D, Lujani B, Chillo O, et al. Overweight, obesity and perceptions about body weight among primary schoolchildren in Dar es Salaam, Tanzania. Tanzan J Health Res. 2014;16(4):304-11 Available from: http://www.ncbi.n/m.nih.gov/pubmed/26 891520. Cited 2019 May 19.

48. Navti LK, Ferrari U, Tange E, Parhofer KG, Pozza SB-D. Height-obesity relationship in school children in Sub-Saharan Africa: results of a crosssectional study in Cameroon. BMC Res Notes. 2015;8(1):98 Available from: http://www.biomedcentral.com/1756-0500/8/98. Cited 2019 May 19.

49. Keino S, Plasqui $G$, Ettyang $G$, van den Borne B. Determinants of stunting and overweight among young children and adolescents in sub-Saharan Africa. Food Nutr Bull. 2014;35(2):167-78 Available from: http://www.ncbi. nlm.nih.gov/pubmed/25076764. Cited 2019 May 19.

50. Melaku YA, Gill TK, Taylor AW, Appleton SL, Gonzalez-Chica D, Adams R, et al. Trends of mortality attributable to child and maternal undernutrition, overweight/obesity and dietary risk factors of non-communicable diseases in sub-Saharan Africa, 1990-2015: findings from the Global Burden of Disease Study 2015. Public Health Nutr. 2019;22(5):827-40 Available from: https://www.cambridge.org/core/product/identifier/S1368980018002975/ type/journal_article. Cited 2019 May 20.

51. Monyeki MA, Awotidebe A, Strydom GL, De Hans RJ, Mamabolo RL, Han CG. The challenges of underweight and overweight in South African children: Are we winning or losing the battle? A systematic review. Int J Environ Res Public Health. 2015;12(2):1156-73 Available from: https://www.mdpi. com/1660-4601/12/2/1156. Cited 2019 May 20.

52. Rossouw HA, Grant CC, Viljoen M. Overweight and obesity in children and adolescents: the south African problem. S Afr J Sci. 2012;108(5-6):31-7.

53. Fruhstorfer $\mathrm{BH}$, Mousoulis $\mathrm{C}$, Uthman OA, Robertson W. Socio-economic status and overweight or obesity among school-age children in subSaharan Africa - a systematic review. Clin Obes. 2016; 6(1): 19-32. Available from: https://doi.org/10.1111/cob.12130.

54. Jaacks LM, Kavle J, Perry A, Nyaku A. Programming maternal and child overweight and obesity in the context of undernutrition: current evidence and key considerations for low- and middle-income countries. Public Health Nutr. 2017;20(7):1286-96 Available from: https://www.cambridge.org/core/ product/identifier/S1368980016003323/type/journal_article.

55. Faye J, Diop M, Gati Ouonkoye R, Seck M, Mandengué SH, Mbengue A, et al. Prevalence of child and teenage obesity in schools in Dakar. Bull Soc Pathol Exot. 2011;104(1):49-52. https://doi.org/10.1007/s13149-010-0101-9 Cited 2019 May 19.

56. Koueta F, Dao L, Dao F, Djekompte S, Sawadogo J, Diarra YE, et al. Factors associated with overweight and obesity among Ouagadougou students (Burkina Faso). Sante. 2011;21(4):227-31 Available from: http://www.ncbi. nlm.nih.gov/pubmed/22407248. Cited 2019 May 19.

57. Kramoh KE, N'goran YNK, Aké-Traboulsi E, Boka BC, Harding DE, Koffi DBJ, et al. Prevalence of obesity in school children in Ivory Coast. Ann Cardiol Angeiol (Paris). 2012;61(3):145-9 Available from: https://linkinghub.elsevier com/retrieve/pii/S0003392812000583. Cited 2019 May 19.

58. Oumar Bâ H, Menta I, Camara Y, Doumbia PS, Diarra MB. Overweight and obesity in the general population of 5-19 years in urban Bamako (Mali). Pan Afr Med J. 2014;19:351 Available from: http://www.ncbi.nlm.nih.gov/ pubmed/25932064. Cited 2019 May 19.

59. Sartorius B, Veerman L, Manyema M, Chola L, Hofman K. Determinants of obesity and associated population attributability, South Africa: Empirical evidence from a national panel survey, 2008-2012. PLoS One. 2015;10(6): e0130218. https://doi.org/10.1371/journal.pone.0130218 Cited 2019 May 20.

60. Tydeman-Edwards R, Van Rooyen FC, Walsh CM. Obesity, undernutrition and the double burden of malnutrition in the urban and rural southern Free State, South Africa. Heliyon. 2018;4(12):e00983 Available from: https:// linkinghub.elsevier.com/retrieve/pii/S240584401833514X. Cited 2019 May 20.

61. Rees A, Thomas N, Brophy S, Knox G, Williams R. Cross sectional study of childhood obesity and prevalence of risk factors for cardiovascular disease and diabetes in children aged 11-13. BMC Public Health. 2009;9(1):86. https://doi.org/10.1186/1471-2458-9-86 Cited 2019 May 20.

62. Adamo KB, Sheel AW, Onywera V, Waudo J, Boit M, Tremblay MS. Child obesity and fitness levels among Kenyan and Canadian children from urban and rural environments: A KIDS-CAN Research Alliance Study. Int J Pediatr Obes. 2011;6(2-2):e225-32. https://doi.org/10.3109/17477166.2010.543683 Cited 2019 May 19.

63. Armstrong MEG, Lambert MI, Lambert EV. Secular trends in the prevalence of stunting, overweight and obesity among South African children (19942004). Eur J Clin Nutr. 2011;65(7):835-40 Available from: http://www.nature. com/articles/ejcn201146. Cited 2019 May 19.

64. Armstrong MEG, Lambert MI, Lambert EV. Relationships between different nutritional anthropometric statuses and health-related fitness of South African primary school children. Ann Hum Biol. 2017;44(3):208-13. https:// doi.org/10.1080/03014460.2016.1224386 Cited 2019 May 19.

65. Craig E, Reilly J, Bland R. Body fatness or anthropometry for assessment of unhealthy weight status? Comparison between methods in South African children and adolescents. Public Health Nutr. 2013;16(11):2005-13 Available from: https://www.cambridge.org/core/product/identifier/S1368980012 004338/type/journal_article. Cited 2019 May 19.

66. Kimani-Murage EW, Kahn K, Pettifor JM, Tollman SM, Klipstein-Grobusch K, Norris SA. Predictors of adolescent weight status and central obesity in rural South Africa. Public Health Nutr. 2011;14(6):1114-22 Available from: https:// www.cambridge.org/core/product/identifier/S1368980011000139/type/ journal_article. Cited 2019 May 19.

67. Ngwenya NA, Ramukumba TS. Prevalence of adolescent obesity at a high school in the City of Tshwane. Curationis. 2017;

68. Negash S, Agyemang C, Matsha TE, Peer N, Erasmus RT, Kengne AP. Differential prevalence and associations of overweight and obesity by gender and population group among school learners in South Africa: A cross-sectional study. BMC Obes. 2017;4(1):29. https://doi.org/10.1186/ s40608-017-0165-1 Cited 2019 May 19.

69. Pretorius SS, Neophytou N, Watson ED. Anthropometric profiles of 8-11 year old children from a low-income setting in South Africa. BMC Public Health. 2019;19(1):314. https://doi.org/10.1186/s12889-019-6530-x Cited 2019 May 19

70. Adegoke S, Olowu W, Adeodu O, Elusiyan J, Dedeke I. Prevalence of overweight and obesity among children in Ile-ife, South-Western Nigeria. West Afr J Med. 2009;28:216-21.

71. Omuemu VO, Omuemu CE. The prevalence of overweight and its risk factors among adolescents in an urban city in Edo State. Niger J Clin Pract. 2010;13(2):128-33 Available from: http://www.ncbi.nlm.nih.gov/pubmed/204 99742. Cited 2019 May 19.

72. Opara D, Ikpeme E, Ekanem U. Prevalence of Stunting, Underweight and Obesity School Aged CHildren in Uyo, Nigeria. Pakistan J Nutr. 2010;9(5): 459-66 Available from: http://docsdrive.com/pdfs/ansinet/pjn/2010/459-466. pdf. Cited 2019 May 20.

73. Senbanjo IO, Oshikoya KA. Physical activity and body mass index of school children and adolescents in Abeokuta, Southwest Nigeria. World J Pediatr. 2010; 6(3):217-22. https://doi.org/10.1007/s12519-010-0209-9 Cited 2019 May 19.

74. Fetuga MB, Ogunlesi TA, Adekanmbi AF, Alabi AD. Growth pattern of schoolchildren in Sagamu, Nigeria using the CDC standards and 2007 WHO standards. Indian Pediatr. 2011;48(7):523-8 Available from: http://www.ncbi. nlm.nih.gov/pubmed/21169645. Cited 2019 May 19.

75. Fetuga MB, Ogunlesi TA, Adekanmbi AF, Alabi AD. Nutritional status of semi-urban Nigerian school children using the 2007 WHO reference population. West Afr J Med. 30(5):331-6 Available from: http://www.ncbi. nlm.nih.gov/pubmed/22752820. Cited 2019 May 19.

76. Adesina AF, Peterside $O$, Anochie I, Akani NA. Weight status of adolescents in secondary schools in port Harcourt using Body Mass Index (BMI). Ital J Pediatr. 2012;38(1):31. https://doi.org/10.1186/1824-7288-38-31 Cited 2019 May 19.

77. Senbanjo 1O, Oshikoya KA. Obesity and blood pressure levels of adolescents in Abeokuta, Nigeria. Cardiovasc J Afr. 2012;23(5):260-4 Available from: http:// www.cvja.co.za/onlinejournal/vol23/vol23_issue5/\#/26/. Cited 2019 May 20.

78. Musa DI, Toriola AL, Monyeki MA, Lawal B. Prevalence of childhood and adolescent overweight and obesity in Benue State, Nigeria. Trop Med Int Health. 2012;17(11):1369-75. https://doi.org/10.1111/j.1365-3156.2012.03083. x Cited 2019 May 20.

79. Maruf FA, Aronu U, Chukwuegbu K, Aronu AE. Influence of gender on prevalence of overweight and obesity in Nigerian schoolchildren and adolescents. Tanzan J Health Res. 2013;15(4):247-51 Available from: http:// www.ncbi.nlm.nih.gov/pubmed/26591700. Cited 2019 May 19. 
80. Omisore B, Omisore AG, Abioye-Kuteyi EA. Gender comparisons of adolescents' anthropometry and blood pressure in Osun State, SouthWestern Nigeria. Int J Adolesc Med Health. 2015;27(3):247-51 Available from: https://www.degruyter.com/view/j/ijamh.2015.27.issue-3/ijamh-20140018/ijamh-2014-0018.xml. Cited 2019 May 20.

81. Okagua J, Alex-Hart BA, Jaja TP. Overweight and obesity status of school adolescents in Portharcourt, southern Nigeria. Niger J Med. 25(1):53-9 Available from: http://www.ncbi.nlm.nih.gov/pubmed/29963820. Cited 2019 May 19.

82. Mekonnen T, Tariku A, Abebe SM. Overweight/obesity among school aged children in Bahir Dar City: cross sectional study. Ital J Pediatr. 2018:44(1):17. https://doi.org/10.1186/s13052-018-0452-6 Cited 2019 May 19.

83. Moges T, Gebremichael B, Shiferaw S, Yirgu R. Is inadequate play area in schools associated with overweight among students in Addis Ababa, Ethiopia? A comparative cross-sectional study. Epidemiol Health. 2018;40: e2018017. https://doi.org/10.4178/epih.e2018017 Cited 2019 May 19.

84. Sorrie MB, Yesuf ME, GebreMichael TG. Overweight/Obesity and associated factors among preschool children in Gondar City, Northwest Ethiopia: A cross-sectional study. PLoS One. 2017;12(8):e0182511. https://doi.org/10. 1371/journal.pone.0182511 Cited 2019 May 19.

85. Tadesse Y, Derso T, Alene KA, Wassie MM. Prevalence and factors associated with overweight and obesity among private kindergarten school children in Bahirdar Town, Northwest Ethiopia: cross-sectional study. BMC Res Notes. 2017;10(1):22. https://doi.org/10.1186/s13104-016-2308-8 Cited 2019 May 19.

86. Wakayo T, Whiting SJ, Belachew T. Vitamin D Deficiency is Associated with Overweight and/or Obesity among Schoolchildren in Central Ethiopia: A Cross-Sectional Study. Nutrients. 2016;8(4):190 Available from: http://www. mdpi.com/2072-6643/8/4/190. Cited 2019 May 19.

87. Teshome T, Singh P, Moges D. Prevalence and Associated Factors of Overweight and Obesity Among High School Adolescents in Urban Communities of Hawassa, Southern Ethiopia. Curr Res Nutr Food Sci J. 2013; 1(1):23-36 Available from: http://www.foodandnutritionjournal.org/volume1 number1/prevalence-and-associated-factors-of-overweight-and-obesityamong-high-school-adolescents-in-urban-communities-of-hawassasouthern-ethiopia/. Cited 2019 May 20.

88. Mosha TC, Fungo S. Prevalence of overweight and obesity among children. Tanzan J Health Res. 2010;12(1):6-16 Available from: http://www.ncbi.nlm. nih.gov/pubmed/20737824. Cited 2019 May 19.

89. Mwaikambo SA, Leyna GH, Killewo J, Simba A, Puoane T. Why are primary school children overweight and obese? A cross sectional study undertaken in Kinondoni district, Dar-es-salaam. BMC Public Health. 2015;15(1):1269 Available from: http://www.biomedcentral.com/1471-2458/15/1269. Cited 2019 May 20.

90. Kimani-Murage EW, Muthuri SK, Oti SO, Mutua MK, van de Vijver S, Kyobutungi C. Evidence of a Double Burden of Malnutrition in Urban Poor Settings in Nairobi, Kenya. PLoS One. 2015;10(6):e0129943. https://doi.org/ 10.1371/journal.pone.0129943 Cited 2019 May 19.

91. Wachira L-JM, Muthuri SK, Ochola SA, Onywera VO, Tremblay MS. Screenbased sedentary behaviour and adiposity among school children: Results from International Study of Childhood Obesity, Lifestyle and the Environment (ISCOLE) - Kenya. PLoS One. 2018;13(6):e0199790. https://doi. org/10.1371/journal.pone.0199790 Cited 2019 May 19.

92. Wamba PCF, Enyong Oben J, Cianflone K. Prevalence of Overweight, Obesity, and Thinness in Cameroon Urban Children and Adolescents. J Obes. 2013;2013:1-9 Available from: http://downloads.hindawi.com/journals/ jobes/2013/737592.pdf. Cited 2019 May 19.

93. Choukem SP, Kamdeu-Chedeu J, Leary SD, Mboue-Djieka Y, Nebongo DN Akazong $C$, et al. Overweight and obesity in children aged 3-13 years in urban Cameroon: A crosssectional study of prevalence and association with socio-economic status. BMC Obes. 2017;4(1):7.

94. Navti LK, Ferrari U, Tange E, Bechtold-Dalla Pozza S, Parhofer KG. Contribution of socioeconomic status, stature and birth weight to obesity in Sub-Saharan Africa: cross-sectional data from primary school-age children in Cameroon. BMC Public Health. 2014;14(1):320. https://doi.org/10.1186/14712458-14-320 Cited 2019 May 19.

95. Mohammed H, Vuvor F. Prevalence of childhood overweight/obesity in basic school in Accra. Ghana Med J. 2012;46(3):124-7 Available from: http:// www.ncbi.nlm.nih.gov/pubmed/23661824. Cited 2019 May 19.

96. Adom T, De Villiers A, Puoane T, Kengne AP. Prevalence and correlates of overweight and obesity among school children in an urban district in Ghana. BMC Obes. 2019;6(1):14. https://doi.org/10.1186/s40608-019-0234-8 Cited 2019 May 19.
97. Kumah DB, Akuffo KO, Abaka-Cann JE, Affram DE, Osae EA. Prevalence of Overweight and Obesity among Students in the Kumasi Metropolis. J Nut Metab. 2015;2015:1-4 Available from: https://www.hindawi.com/journals/ jnme/2015/613207/abs/. Cited 2019 May 20.

98. dos Santos FK, Maia JAR, Gomes TNQF, Daca T, Madeira A, Katzmarzyk PT, et al. Secular trends in growth and nutritional status of Mozambican schoolaged children and adolescents. PLoS One. 2014;9(12):e114068. https://doi. org/10.1371/journal.pone.0114068 Cited 2019 May 19.

99. Dos Santos FK, Prista A, Gomes TNQF, Santos D, Damasceno A, Madeira A, et al. Body mass index, cardiorespiratory fitness and cardiometabolic risk factors in youth from Portugal and Mozambique. Int J Obes. 2015;39(10): 1467-74 Available from: http://www.nature.com/articles/ijo2015110. Cited 2019 May 20

100. Nagwa MA, Elhussein AM, Azza M, Abdulhadi NH. Alarming high prevalence of overweight/obesity among Sudanese children. Eur J Clin Nutr. 2011;65(3): 409-11 Available from: http://www.nature.com/articles/ejcn2010253. Cited 2019 May 19.

101. Salman Z, Kirk GD, Deboer MD. High rate of Obesity-associated hypertension among primary schoolchildren in Sudan. Int J Hypertens. 2011;2011:1-6.

102. Christoph MJ, Grigsby-Toussaint DS, Baingana R, Ntambi JM. Physical Activity, Sleep, and BMI Percentile in Rural and Urban Ugandan Youth. Ann Glob Heal. 2017;83(2):311-9. https://doi.org/10.1016/j.aogh.2017.04.005/ Cited 2019 May 19.

103. Wrotniak BH, Malete L, Maruapula SD, Jackson J, Shaibu S, Ratcliffe S, et al. Association between socioeconomic status indicators and obesity in adolescent students in Botswana, an African country in rapid nutrition transition. Pediatr Obes. 2012;7(2):e9-13. https://doi.org/10.1111/j.2047-6310. 2011.00023.x Cited 2019 May 19.

104. Juwara A, Huang N, Chien L-Y, Chen H-J. Stunting and weight statuses of adolescents differ between public and private schools in urban Gambia. Int J Public Health. 2016;61(6):717-26. https://doi.org/10.1007/s00038-016-08366 Cited 2019 May 19.

105. van den Berg VL, Seheri L, Raubenheimer J. Body mass index of 16-year olds in urban Maseru, Lesotho. African J Prim Heal Care Fam Med. 2014;6(1): E1-14 Available from: https://phcfm.org/index.php/phcfm/article/view/618. Cited 2019 May 19.

106. Caleyachetty R, Rudnicka AR, Echouffo-Tcheugui JB, Siegel KR, Richards N, Whincup PH. Prevalence of overweight, obesity and thinness in 9-10 year old children in Mauritius. Global Health. 2012;8(1):28. https://doi.org/10. 1186/1744-8603-8-28 Cited 2019 May 20.

107. Bovet P, Chiolero A, Madeleine G, Paccaud F. Prevalence of overweight and underweight in public and private schools in the Seychelles. Int J Pediatr Obes. 2010;5:274-8.

108. Sagbo H, Ekouevi DK, Ranjandriarison DT, Niangoran S, Bakai TA, Afanvi A, et al. Prevalence and factors associated with overweight and obesity among children from primary schools in urban areas of Lomé, Togo. Public Health Nutr. 2018;21(6):1048-56 Available from: https://www.cambridge.org/core/ product/identifier/S1368980017003664/type/journal_article. Cited 2019 May 20.

109. Kambondo G, Sartorius B. Risk Factors for Obesity and Overfat among Primary School Children in Mashonaland West Province, Zimbabwe. Int J Environ Res Public Health. 2018;15(2):249 Available from: http://www.mdpi. com/1660-4601/15/2/249. Cited 2019 May 19.

110. Muthuri SK, Onywera VO, Tremblay MS, Broyles ST, Chaput J-P, Fogelholm $M$, et al. Relationships between Parental Education and Overweight with Childhood Overweight and Physical Activity in 9-11 Year Old Children: Results from a 12-Country Study. PLoS One. 2016;11(8):e0147746. https://doi. org/10.1371/journal.pone.0147746 Cited 2019 May 20.

111. Manyanga T, El-Sayed H, Doku DT, Randall JR. The prevalence of underweight, overweight, obesity and associated risk factors among schoolgoing adolescents in seven African countries. BMC Public Health. 2014;14(1): 887. https://doi.org/10.1186/1471-2458-14-887 Cited 2019 May 20.

112. Kruger HS, Steyn NP, Swart EC, Maunder EMW, Nel JH, Moeng L, et al. Overweight among children decreased, but obesity prevalence remained high among women in South Africa, 1999-2005. Public Health Nutr. 2011; 15(4):594-9.

113. Reddy SP, Resnicow K, James S, Funani IN, Kambaran NS, Omardien RG, et al. Rapid increases in overweight and obesity among South African adolescents: comparison of data from the South African National Youth Risk Behaviour Survey in 2002 and 2008. Am J Public Health. 2012;102(2):262-8. https://doi.org/10.2105/AJPH.2011.300222 Cited 2019 May 19. 
114. Senbanjo IO, Oshikoya KA, Njokanma OF. Changes in the nutritional status of school children and adolescents in Abeokuta, Nigeria between 1983 and 2006 Les changements dans l'etat nutritionnel des enfants d'age scolaire et des adolescents dans Abeokuta, au Nigeria entre 1983 et 2006. West Afr J Med. 2011;30(6):425-31 Available from: http://www.ajol.info/index.php/ wajm/article/download/98493/87765 http://ovidsp.ovid.com/ovidweb.cgi?T= $J S \& C S C=Y \& N E W S=N \& P A G E=$ fulltext\&D=emed $11 \& A N=2013785342 \mathrm{http}: / / \mathrm{bf} 4$ dv7zn3u.search.serialssolutions.com.myaccess.library.utoronto.ca/?url_ver=Z3 9.88-2004\&rft. Cited 2019 May 19.

115. Kimani-Murage EW, Kahn K, Pettifor JM, Tollman SM, Dunger DB, GómezOlivé XF, et al. The prevalence of stunting, overweight and obesity, and metabolic disease risk in rural South African children. BMC Public Health. 2010;10:158

116. Meko LNM, Slabber-Stretch M, Walsh CM, Kruger SH, Nel M. School environment, socioeconomic status and weight of children in Bloemfontein, South Africa. African J Prim Heal Care Fam Med. 2015;7(1) Available from: https://phcfm.org/index.php/phcfm/article/view/751. Cited 2019 May 19.

117. Moselakgomo VK, van Staden M. Diagnostic comparison of Centers for Disease Control and Prevention and International Obesity Task Force criteria for obesity classification in South African children. African J Prim Heal Care Fam Med. 2017;9(1):e1-7 Available from: https://phcfm.org/index.php/ phcfm/article/view/1383. Cited 2019 May 19.

118. Sedibe MH, Pisa PT, Feeley AB, Pedro TM, Kahn K, Norris SA. Dietary habits and eating practices and their association with overweight and obesity in rural and urban black South African adolescents. Nutrients. 2018;10(2):145 Available from: http://www.mdpi.com/2072-6643/10/2/145. Cited 2019 May 19.

119. Zeelie A, Moss SJ, Kruger HS. The relationship between body composition and selected metabolic syndrome markers in black adolescents in South Africa: The PLAY study. Nutrition. 2010;26(11-12):1059-64 Available from: https:// linkinghub.elsevier.com/retrieve/pii/S0899900710000961. Cited 2019 May 19.

120. Akodu SO, Diaku-Akinwumi IN, Njokanma OF. Obesity--does it occur in Nigerian children with sickle cell anemia. Pediatr Hematol Oncol. 2012;29(4): 358-64. https://doi.org/10.3109/08880018.2012.680682 Cited 2019 May 19.

121. Ene-Obong H, Ibeanu V, Onuoha N, Ejekwu A. Prevalence of overweight, obesity, and thinness among urban school-aged children and adolescents in southern Nigeria. Food Nutr Bull. 2012;33(4):242-50 Available from: http:// www.ncbi.nlm.nih.gov/pubmed/23424890. Cited 2019 May 19

122. Nwaiwu O, Ibe BC. Relationship between serum cholesterol and body mass index in Nigeria schoolchildren aged 2-15 years. J Trop Pediatr. 2015;61(2): 126-30. https://doi.org/10.1093/tropej/fmu080 Cited 2019 May 19.

123. Oduwole AA, Ladapo TA, Fajolu IB, Ekure EN, Adeniyi OF. Obesity and elevated blood pressure among adolescents in Lagos, Nigeria: a crosssectional study. BMC Public Health. 2012;12(1):616. https://doi.org/10.1186/ 1471-2458-12-616 Cited 2019 May 19.

124. Uwaezuoke SN, Okoli CV, Ubesie AC, Ikefuna AN. Primary hypertension among a population of Nigerian secondary school adolescents: Prevalence and correlation with anthropometric indices: A cross-sectional study. Niger J Clin Pract. 2016;19(5):649-54 Available from: http://www.njcponline.com/ text.asp?2016/19/5/649/188706. Cited 2019 May 19.

125. Mushengezi $B$, Chillo P. Association between body fat composition and blood pressure level among secondary school adolescents in Dar es Salaam, Tanzania. Pan Afr Med J. 2014:19 Available from: http://www.panafricanmed-journal.com/content/article/19/327/full. Cited 2019 May 20.

126. Lesiapeto M, Smuts C, Hanekom S, Du Plessis J, Faber M. Risk factors of poor anthropometric status in children under five years of age living in rural districts of the Eastern Cape and KwaZulu-Natal provinces, South Africa. South African J Clin Nutr. 2016;23(4):202-7 Available from: https://www.ajol. info/index.php/sajcn/article/view/63118. Cited 2019 May 20.

127. Lundeen EA, Norris SA, Adair LS, Richter LM, Stein AD. Sex differences in obesity incidence: 20-year prospective cohort in South Africa. Pediatr Obes. 2016;11(1):75-80. https://doi.org/10.1111/ijpo.12039 Cited 2019 May 19.

128. Peltzer K, Pengpid S. Overweight and obesity and associated factors among school-aged adolescents in Ghana and Uganda. Int J Environ Res Public Health. 2011;8(10):3859-70 Available from: http://www.mdpi.com/1660-46 01/8/10/3859. Cited 2019 May 19.

129. Steyn HNP, Labadarios BD, Nel SJ, Kruger HS, Maunder E. What is the nutritional status of children of obese mothers in South Africa? Nutrition. 2011;27(9):904-11. Available from:. https://doi.org/10.1016/j.nut.2010.10.007.

130. Symington EA, Gericke GJ, Nel JH, Labadarios D. The relationship between stunting and overweight among children from South Africa: Secondary analysis of the national food consumption survey - Fortification baseline I.
South African Med J. 2016;106(1):65-9 Available from: https://www.ajol.info/ index.php/samj/article/view/131158. Cited 2019 May 20.

131. Tathiah N, Moodley I, Mubaiwa V, Denny L, Taylor M. South Africa's nutritional transition: Overweight, obesity, underweight and stunting in female primary school learners in rural KwaZulu-Natal, South Africa. South African Med J. 2013;103(10):718-23 Available from: http://www.samj.org.za/ index.php/samj/article/view/6922. Cited 2019 May 20.

132. Tchoubi S, Sobngwi-Tambekou J, JJN N, Asangbeh SL, Nkoum BA, Sobngwi E. Prevalence and Risk Factors of Overweight and Obesity among Children Aged 6-59 Months in Cameroon: A Multistage, Stratified Cluster Sampling Nationwide Survey. PLoS One. 2015;10(12):e0143215. https://doi.org/10. 1371/journal.pone.0143215 Cited 2019 May 19.

133. Turi KN, Christoph MJ, Grigsby-Toussaint DS. Spatial distribution of underweight, overweight and obesity among women and children: results from the 2011 Uganda Demographic and Health Survey. Int J Environ Res Public Health. 2013;10(10):4967-81 Available from: http://www.mdpi. com/1660-4601/10/10/4967. Cited 2019 May 19.

134. Feeley AB, Musenge $E$, Pettifor JM, Norris SA. Investigation into longitudinal dietary behaviours and household socio-economic indicators and their association with BMI Z-score and fat mass in South African adolescents: the Birth to Twenty (Bt20) cohort. Public Health Nutr. 2013;16(4):693-703 Available from: https://www.cambridge.org/core/journals/public-healthnutrition/article/investigation-into-longitudinal-dietary-behaviours-andhousehold-socioeconomic-indicators-and-their-association-with-bmi-zscoreand-fat-mass-in-south-african-adolescents-the-birth-to-. Cited 2019 May 20.

135. Ginsburg C, Griffiths PL, Richter LM, Norris SA. Residential mobility, socioeconomic context and body mass index in a cohort of urban South African adolescents. Heal Place. 2013;19(1):99-107 Available from: https:// linkinghub.elsevier.com/retrieve/pii/S1353829212001827. Cited 2019 May 20.

136. Munthali RJ, Kagura J, Lombard Z, Norris SA. Childhood adiposity trajectories are associated with late adolescent blood pressure: birth to twenty cohort. BMC Public Health. 2016;16(1):665. https://doi.org/10.1186/ s12889-016-3337-x Cited 2019 May 19.

137. Gewa CA. Childhood overweight and obesity among Kenyan pre-school children: association with maternal and early child nutritional factors. Public Health Nutr. 2010;13(4):496-503 Available from: https://www.cambridge. org/core/product/identifier/S136898000999187X/type/journal_article. Cited 2019 May 19

138. Baumgartner J, Smuts CM, Aeberli I, Malan L, Tjalsma H, Zimmermann MB. Overweight impairs efficacy of iron supplementation in iron-deficient South African children: A randomized controlled intervention. Int J Obes. 2013;37(1):24-30. https://doi.org/10.1038/ijo.2012.145 Cited 2019 May 20.

139. Mokabane NN, Mashao MM, Van Staden M, Potgieter M, Potgieter A. Low levels of physical activity in female adolescents cause overweight and obesity: Are our schools failing our children? South African Med J. 2014; 104(10):665 Available from: http://www.samj.org.za/index.php/samj/article/ view/8577. Cited 2019 May 19.

140. Pan American Health Organization. The economic burden of noncommunicable diseases in the Americas. Econ Burd Non-Communicable Dis Am. 2017..

141. WHO, Regional Office for Africa. Obesity1. WHO Regional Office for Africa. Obesity: WHO; 2017. Available from: https://www.afro.who.int/health-topics/ obesity. Cited 2019 May 3.

142. Ng M, Fleming T, Robinson M, Thomson B, Graetz N, Margono C, et al. Global, regional, and national prevalence of overweight and obesity in children and adults during 1980-2013: a systematic analysis for the Global Burden of Disease Study 2013. Lancet. 2014;384(9945):766-81 Available from: https://linkinghub.elsevier.com/retrieve/pii/S0140673614604608. Cited 2019 May 19.

143. Bollyky BTJ, Templin T, Cohen M, Dieleman JL. Lower-Income Countries That Face The Most Rapid Shift In Noncommunicable Disease Burden Are Also The Least Prepared. Health Aff (Millwood). 2017;11(11):1866-75

144. World Bank Data Team. New country classifications by income level: 20192020. 2019. Available from: http://blogs.worldbank.org/opendata/newcountry-classifications-income-level-2019-2020. Cited 2020 Jan 21.

145. UNICEF, WHO, World Bank Group. Levels and Trends in Child Malnutrition: UNICEF, WHO, Wold Bank Group joint child malnutrition estimates. 2016. Available from: www.who.int/nutgrowthdb/estimates. Cited 2019 May 3. 
146. Duncan S, Duncan EK, Fernandes RA, Buonani C, Bastos KD-N, Segatto AF, et al. Modifiable risk factors for overweight and obesity in children and adolescents from São Paulo, Brazil. BMC Public Health. 2011;11(1):585. https://doi.org/10.1186/1471-2458-11-585 Cited 2019 May 6.

147. OECD/EU. Obesity Update 2017. OECD Updat Rep. 2017;13(5):331-41 Available from: www.oecd.org/health/obesity-update.htm.

148. Tzioumis E, Adair LS. Childhood dual burden of under-and overnutrition in low-and middle-income countries: a critical review. Food Nutr Bull. 35(2): 230-43. https://doi.org/10.1177/156482651403500210 journals.sagepub.com. Cited 2019 May 20.

\section{Publisher's Note}

Springer Nature remains neutral with regard to jurisdictional claims in published maps and institutional affiliations.

Ready to submit your research? Choose BMC and benefit from:

- fast, convenient online submission

- thorough peer review by experienced researchers in your field

- rapid publication on acceptance

- support for research data, including large and complex data types

- gold Open Access which fosters wider collaboration and increased citations

- maximum visibility for your research: over $100 \mathrm{M}$ website views per year

At BMC, research is always in progress.

Learn more biomedcentral.com/submissions 